\title{
Structure Elucidation of (+)-Amphidinolide A by Total Synthesis and NMR Chemical Shift Analysis
}

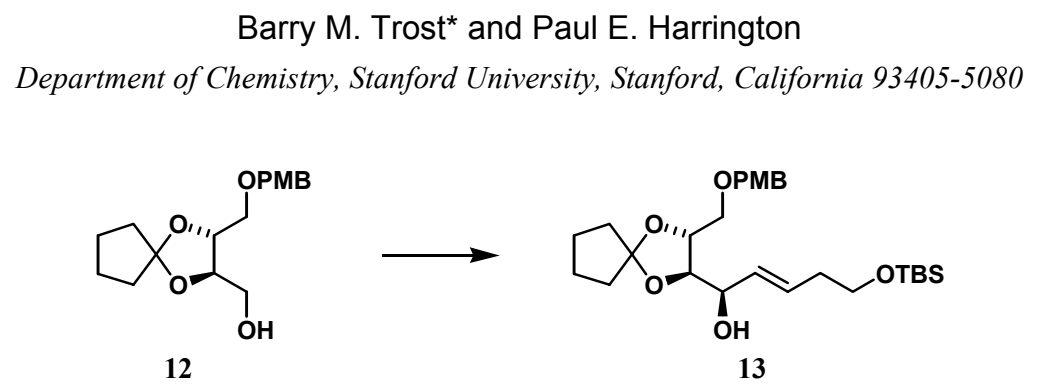

To a solution of oxalyl chloride $(1.50 \mathrm{~mL}, 2.18 \mathrm{~g}, 17.2 \mathrm{mmol})$ in $\mathrm{CH}_{2} \mathrm{Cl}_{2}(40 \mathrm{~mL})$ at -78 ${ }^{\circ} \mathrm{C}$ was added DMSO (2.50 mL, $\left.2.75 \mathrm{~g}, 35.2 \mathrm{mmol}\right)$, stirred for $15 \mathrm{~min}$, and a solution of alcohol $12(3.264 \mathrm{~g}, 10.6 \mathrm{mmol})$ in $\mathrm{CH}_{2} \mathrm{Cl}_{2}(35 \mathrm{~mL})$ at $-78^{\circ} \mathrm{C}$ was added via cannula. After $1 \mathrm{~h}$ at -78 ${ }^{\circ} \mathrm{C}$, triethylamine $(7.4 \mathrm{~mL}, 5.4 \mathrm{~g}, 53 \mathrm{mmol})$ was added, the reaction mixture was stirred at $-78^{\circ} \mathrm{C}$ for $15 \mathrm{~min}$, warmed to room temperature, diluted with $\mathrm{CH}_{2} \mathrm{Cl}_{2}$, washed with saturated $\mathrm{NH}_{4} \mathrm{Cl}$ (2 x), brine ( $1 \mathrm{x}$ ), dried over $\mathrm{MgSO}_{4}$, filtered through a pad of silica gel, and concentrated to give the aldehyde which was used in the next step without further purification: ${ }^{1} \mathrm{H}$ NMR $(500 \mathrm{MHz}$, $\left.\mathrm{CDCl}_{3}\right) \delta 9.75(\mathrm{~d}, J=1.7 \mathrm{~Hz}, 1 \mathrm{H}), 7.26(\mathrm{~d}, J=8.8 \mathrm{~Hz}, 2 \mathrm{H}), 6.88(\mathrm{~d}, J=8.5 \mathrm{~Hz}, 2 \mathrm{H}), 4.53(\mathrm{~s}$, 2H), $4.51(\mathrm{~m}, 1 \mathrm{H}), 4.21(\mathrm{~m}, 1 \mathrm{H}), 4.17(\mathrm{dd}, J=7.1,1.7 \mathrm{~Hz}, 1 \mathrm{H}), 3.81(\mathrm{~s}, 3 \mathrm{H}), 3.79(\mathrm{~m}, 1 \mathrm{H}), 3.62$ (d, $J=4.9 \mathrm{~Hz}, 1 \mathrm{H}), 1.92-1.62(\mathrm{~m}, 8 \mathrm{H})$. To a solution of $\mathrm{HC} \equiv \mathrm{CCH}_{2} \mathrm{CH}_{2} \mathrm{OTBS}(4.849 \mathrm{~g}, 26.3$ mmol) in THF $(100 \mathrm{~mL})$ at $0{ }^{\circ} \mathrm{C}$ was added $n$-BuLi $(10.5 \mathrm{~mL}, 2.5 \mathrm{M}$ in hexanes, $26.3 \mathrm{mmol})$, stirred for $15 \mathrm{~min}$, cooled to $-60^{\circ} \mathrm{C}$, and $\mathrm{ClTi}(\mathrm{O} i-\mathrm{Pr})_{3}(6.25 \mathrm{~mL}, 6.82 \mathrm{~g}, 26.2 \mathrm{mmol})$ was added. The solution was stirred at $-60^{\circ} \mathrm{C}$ for $1.5 \mathrm{~h}$, cooled to $-78{ }^{\circ} \mathrm{C}$, and a solution of the aldehyde, prepared in the previous step, in THF $(80 \mathrm{~mL})$ at $-78^{\circ} \mathrm{C}$ was added via cannula. The reaction mixture was stirred at $-78^{\circ} \mathrm{C}$ for $1 \mathrm{~h}$, warmed from $-78^{\circ} \mathrm{C}$ to $-50^{\circ} \mathrm{C}$ over $1 \mathrm{~h}$, quenched with saturated $\mathrm{NH}_{4} \mathrm{Cl}$, warmed to room temperature, and diluted with ether and water. The aqueous phase was extracted with ether $(6 \mathrm{x})$ and the combined organic extracts were washed with brine $(1 \mathrm{x})$, dried over $\mathrm{MgSO}_{4}$, concentrated, and used in the next step without further purification: ${ }^{1} \mathrm{H}$ NMR (500 MHz, $\left.\mathrm{CDCl}_{3}\right) \delta 7.26(\mathrm{~d}, J=8.8 \mathrm{~Hz}, 2 \mathrm{H}), 6.87(\mathrm{~d}, J=8.8 \mathrm{~Hz}, 2 \mathrm{H}), 4.55-4.52(\mathrm{~m}, 2 \mathrm{H})$, 4.49 (m, 1H), 3.86 (dd, $J=7.3,4.4 \mathrm{~Hz}, 1 \mathrm{H}), 3.81-3.78$ (m, 3H), 3.80 (s, 3H), 3.69 (t, $J=7.3 \mathrm{~Hz}$, 2H), $3.62(\mathrm{~d}, J=5.1 \mathrm{~Hz}, 1 \mathrm{H}), 2.45(\mathrm{~d}, J=4.6 \mathrm{~Hz}, 1 \mathrm{H}), 2.38$ (dddd, $J=7.1,7.1,2.0,2.0 \mathrm{~Hz}, 1 \mathrm{H}$ ), 1.88-1.65 (m, 8H), $0.89(\mathrm{~s}, 9 \mathrm{H}), 0.08(\mathrm{~s}, 6 \mathrm{H})$. To a solution of the alkyne, prepared in the previous step, in THF $(33 \mathrm{~mL})$ at $0{ }^{\circ} \mathrm{C}$ was added Red-Al ${ }^{\circledR}(5.6 \mathrm{~mL}, 65 \%$ wt in toluene, 18.7 
mmol). The reaction mixture was warmed to room temperature, stirred for $6 \mathrm{~h}$, quenched with aqueous potassium sodium tartrate, stirred for $20 \mathrm{~min}$, and diluted with ether and water. The aqueous phase was extracted with $\mathrm{Et}_{2} \mathrm{O}(5 \mathrm{x})$ and the combined organic extracts were washed with brine $(1 \mathrm{x})$, dried over $\mathrm{MgSO}_{4}$, and concentrated. Purification by flash column chromatography on silica gel (20\% to $30 \%$ to $50 \%$ EtOAc in petroleum ether) gave alkene 13 (2.636 g, 51\%) and recovered alcohol $12(747 \mathrm{mg}, 23 \%)$ as colorless oils. Data for 13: $\mathrm{R}_{f}=0.29$ (20\% EtOAc in petroleum ether); ${ }^{1} \mathrm{H}$ NMR $\left(500 \mathrm{MHz}, \mathrm{CDCl}_{3}\right) \delta 7.25(\mathrm{~d}, J=8.8 \mathrm{~Hz}, 2 \mathrm{H}), 6.87$ $(\mathrm{d}, J=8.8 \mathrm{~Hz}, 2 \mathrm{H}), 5.76$ (dddd, $J=13.9,6.8,6.8,1.2 \mathrm{~Hz}, 1 \mathrm{H}), 5.51$ (ddd, $J=15.4,6.1,1.2 \mathrm{~Hz}$, $1 \mathrm{H}), 4.51(\mathrm{~s}, 2 \mathrm{H}), 4.18(\mathrm{~m}, 1 \mathrm{H}), 4.07$ (ddd, $J=7.3,5.4,5.4 \mathrm{~Hz}, 1 \mathrm{H}), 3.80(\mathrm{~s}, 3 \mathrm{H}), 3.70(\mathrm{dd}, J=$ 7.6, $5.6 \mathrm{~Hz}, 1 \mathrm{H}), 3.62(\mathrm{t}, J=6.8 \mathrm{~Hz}, 2 \mathrm{H}), 3.58(\mathrm{dd}, J=10.0,5.6 \mathrm{~Hz}, 1 \mathrm{H}), 3.51$ (dd, $J=10.0,5.1$ $\mathrm{Hz}, 1 \mathrm{H}), 2.57(\mathrm{~d}, J=2.9 \mathrm{~Hz}, 1 \mathrm{H}), 2.25$ (q br, $J=6.8 \mathrm{~Hz}, 2 \mathrm{H}), 1.88-1.62(\mathrm{~m}, 8 \mathrm{H}), 0.89$ (s, 9H), $0.04(\mathrm{~s}, 6 \mathrm{H}) ;{ }^{13} \mathrm{C} \mathrm{NMR}\left(101 \mathrm{MHz}, \mathrm{CDCl}_{3}\right) \delta 159.3,130.1,129.8,129.7,129.4,119.3,113.8$, $81.2,76.7,73.2,72.2,70.4,68.2,55.2,37.2,37.1,36.0,25.9,23.6,23.3,18.3,-5.3$; IR (neat) 3458 (br), 2955, 1614, 1515, 1250, $1102 \mathrm{~cm}^{-1}$; MALDIMS m/z $515.3[\mathrm{M}+\mathrm{Na}]^{+}$; optical rotation $[\alpha]^{27}+9.5^{\circ}\left(c\right.$ 1.0, $\left.\mathrm{CH}_{2} \mathrm{Cl}_{2}\right)$. Anal. Calcd for $\mathrm{C}_{27} \mathrm{H}_{44} \mathrm{O}_{6} \mathrm{Si}: \mathrm{C}, 65.82 ; \mathrm{H}, 9.00$. Found: C, 65.66; H, 8.87 .
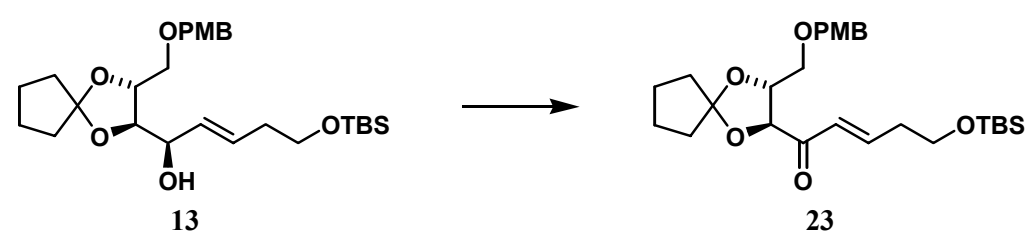

To a solution of alcohol $13(3.038 \mathrm{~g}, 6.17 \mathrm{mmol})$ in $\mathrm{CH}_{2} \mathrm{Cl}_{2}(40 \mathrm{~mL})$ at $0{ }^{\circ} \mathrm{C}$ was added $\mathrm{NaHCO}_{3}(3.668 \mathrm{~g}, 43.7 \mathrm{mmol})$ and Dess-Martin reagent $(3.559 \mathrm{~g}, 8.39 \mathrm{mmol})$. The reaction mixture was warmed to room temperature, stirred for $2 \mathrm{~h}$, quenched with saturated $\mathrm{Na}_{2} \mathrm{SO}_{3}$, stirred for $15 \mathrm{~min}$, and diluted with water. The aqueous phase was extracted with $\mathrm{CH}_{2} \mathrm{Cl}_{2}(5 \mathrm{x})$ and the combined organic extracts were washed with brine $(1 \mathrm{x})$, dried over $\mathrm{MgSO}_{4}$, and concentrated. Purification by flash column chromatography on silica gel $(5 \%$ to $10 \%$ to $15 \%$ EtOAc in petroleum ether) gave ketone $23(2.613 \mathrm{~g}, 86 \%)$ as a colorless oil: $\mathrm{R}_{f}=0.20(10 \%$ EtOAc in petroleum ether); ${ }^{1} \mathrm{H} \mathrm{NMR}\left(400 \mathrm{MHz}, \mathrm{CDCl}_{3}\right) \delta 7.27(\mathrm{~d}, J=8.4 \mathrm{~Hz}, 2 \mathrm{H}), 7.05$ (ddd, $J$ $=15.9,7.2,7.2 \mathrm{~Hz}, 1 \mathrm{H}), 6.87(\mathrm{~d}, J=8.7 \mathrm{~Hz}, 2 \mathrm{H}), 6.58(\mathrm{ddd}, J=15.9,1.5,1.5 \mathrm{~Hz}, 1 \mathrm{H}), 4.54(\mathrm{~s}$, $2 \mathrm{H}), 4.30(\mathrm{~d}, J=7.0 \mathrm{~Hz}, 1 \mathrm{H}), 4.23(\mathrm{ddd}, J=7.0,5.8,3.5 \mathrm{~Hz}, 1 \mathrm{H}), 3.80(\mathrm{~s}, 3 \mathrm{H}), 3.73(\mathrm{t}, J=6.4$ $\mathrm{Hz}, 2 \mathrm{H}), 3.70(\mathrm{dd}, J=10.7,3.5 \mathrm{~Hz}, 1 \mathrm{H}), 3.60(\mathrm{dd}, J=10.5,5.6 \mathrm{~Hz}, 1 \mathrm{H}), 2.44(\mathrm{ddd}, J=13.6,6.6$, 
$1.5 \mathrm{~Hz}, 2 \mathrm{H}), 1.91-1.67(\mathrm{~m}, 8 \mathrm{H}), 0.88(\mathrm{~s}, 9 \mathrm{H}), 0.04(\mathrm{~s}, 6 \mathrm{H}) ;{ }^{13} \mathrm{C} \mathrm{NMR}\left(101 \mathrm{MHz}, \mathrm{CDCl}_{3}\right) \delta 197.7$, 159.2, 147.1, 130.0, 129.4, 126.5, 120.9, 113.8, 80.8, 77.5, 73.2, 69.9, 61.4, 55.3, 36.8, 36.7, 36.2, 25.9, 23.7, 23.3, 18.3, -5.3; IR (neat) 2959, 1691, 1252, 1103, $836 \mathrm{~cm}^{-1}$; EIMS m/z 277 (1), 213 (1), 145 (1), 137 (1), 121 (100); HREIMS calcd for $\mathrm{C}_{27} \mathrm{H}_{42} \mathrm{O}_{6} \mathrm{Si}$ 490.2751, found 490.2751; optical rotation $[\alpha]^{27}{ }_{D}+6.0^{\circ}\left(c\right.$ 1.0, $\left.\mathrm{CH}_{2} \mathrm{Cl}_{2}\right)$. Anal. Calcd for $\mathrm{C}_{27} \mathrm{H}_{42} \mathrm{O}_{6} \mathrm{Si}: \mathrm{C}, 66.09 ; \mathrm{H}, 8.63$. Found: C, 66.40; H, 8.62.
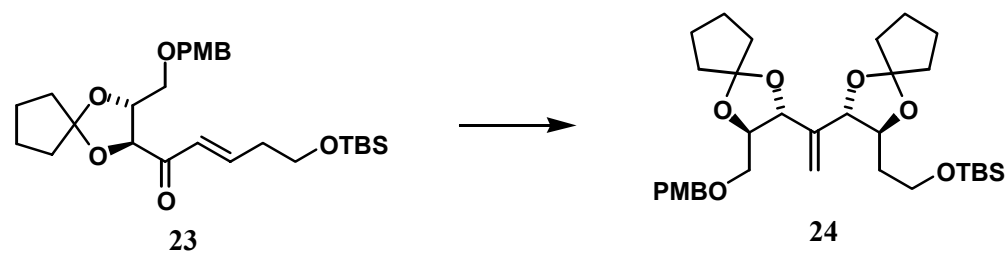

To a mixture of $\mathrm{K}_{3} \mathrm{Fe}(\mathrm{CN})_{6}(17.96 \mathrm{~g}, 54.5 \mathrm{mmol}), \mathrm{K}_{2} \mathrm{CO}_{3}(7.57 \mathrm{~g}, 54.8 \mathrm{mmol}), \mathrm{NaHCO}_{3}$ (4.62 g, $55.0 \mathrm{mmol}$ ), methanesulfonamide (1.720 g, $18.1 \mathrm{mmol}$ ), and (DHQ) 2 -PHAL (369 mg, $0.47 \mathrm{mmol})$ at room temperature was added water $(85 \mathrm{~mL})$ and tert-butyl alcohol $(45 \mathrm{~mL})$. The reaction mixture was cooled to $0{ }^{\circ} \mathrm{C}, \mathrm{K}_{2} \mathrm{OsO}_{4} \cdot 2 \mathrm{H}_{2} \mathrm{O}(171 \mathrm{mg}, 0.46 \mathrm{mmol})$ was added followed by a solution of alkene $23(8.871 \mathrm{~g}, 18.1 \mathrm{mmol})$ in tert-butyl alcohol (40 mL). The reaction mixture was stirred at $0{ }^{\circ} \mathrm{C}$ for $22 \mathrm{~h}$, quenched with $\mathrm{Na}_{2} \mathrm{SO}_{3}(80 \mathrm{~g})$, warmed to room temperature, stirred for $1 \mathrm{~h}$, and diluted with EtOAc and water. The aqueous phase was extracted with EtOAc (4 x) and the combined organic extracts were washed with $2 \mathrm{M} \mathrm{NaOH}(1 \mathrm{x})$, brine $(1 \mathrm{x})$, dried over $\mathrm{MgSO}_{4}$, and concentrated to give the diol which was used in the next step without further purification: $\mathrm{R}_{f}=0.46$ (30\% EtOAc in petroleum ether); ${ }^{1} \mathrm{H}$ NMR $\left(500 \mathrm{MHz}, \mathrm{CDCl}_{3}\right) \delta 7.27(\mathrm{~d}, J$ $=7.8 \mathrm{~Hz}, 2 \mathrm{H}), 6.87(\mathrm{~d}, J=8.5 \mathrm{~Hz}, 2 \mathrm{H}), 4.55(\mathrm{~s}, 2 \mathrm{H}), 4.52(\mathrm{~d} \mathrm{br}, J=7.3 \mathrm{~Hz}, 1 \mathrm{H}), 4.48(\mathrm{~d}, J=6.8$ $\mathrm{Hz}, 1 \mathrm{H}), 4.34$ (ddd, $J=5.9,5.9,3.2 \mathrm{~Hz}, 1 \mathrm{H}), 3.95-3.85$ (m, 3H), 3.80 (s, 3H), 3.75 (dd, $J=11.0$, $3.2 \mathrm{~Hz}, 1 \mathrm{H}), 3.62(\mathrm{dd}, J=10.8,5.9 \mathrm{~Hz}, 1 \mathrm{H}), 3.45$ (d, $J=6.8 \mathrm{~Hz}, 1 \mathrm{H}), 3.14(\mathrm{~d}, J=4.9 \mathrm{~Hz}, 1 \mathrm{H})$, 2.03-1.66 (m, 10H), $0.89(\mathrm{~s}, 9 \mathrm{H}), 0.08(\mathrm{~s}, 6 \mathrm{H})$. To a solution of the diol, prepared in the previous step, and 1,1-dimethoxycyclopentane $(25 \mathrm{~mL})$ in $\mathrm{CH}_{2} \mathrm{Cl}_{2}(190 \mathrm{~mL})$ at $0{ }^{\circ} \mathrm{C}$ was added $\mathrm{TsOH} \cdot \mathrm{H}_{2} \mathrm{O}$ (562 mg, $2.95 \mathrm{mmol}$ ). The reaction mixture was stirred at $0{ }^{\circ} \mathrm{C}$ for $2 \mathrm{~h}$, diluted with ether, washed with saturated $\mathrm{NaHCO}_{3}(1 \mathrm{x})$, brine $(1 \mathrm{x})$, dried over $\mathrm{MgSO}_{4}$, and concentrated to give ketal 14 which was used in the next step without further purification: $\mathrm{R}_{f}=0.68(30 \%$ EtOAc in petroleum ether); ${ }^{1} \mathrm{H}$ NMR $\left(500 \mathrm{MHz}, \mathrm{CDCl}_{3}\right) \delta 7.27(\mathrm{~d}, J=8.8 \mathrm{~Hz}, 2 \mathrm{H}), 6.87(\mathrm{~d}, J=8.5 \mathrm{~Hz}, 2 \mathrm{H}), 4.57-$ $4.51(\mathrm{~m}, 3 \mathrm{H}), 4.41(\mathrm{~d}, J=7.1 \mathrm{~Hz}, 1 \mathrm{H}), 4.26(\mathrm{ddd}, J=9.3,6.1,3.2 \mathrm{~Hz}, 1 \mathrm{H}), 4.18(\mathrm{ddd}, J=8.8$, 
7.1, 3.7 Hz, 1H), 3.80 (s, 3H), 3.79-3.72 (m, 3H), 3.62 (dd, $J=10.7,6.1 \mathrm{~Hz}, 1 \mathrm{H}), 2.07-1.63$ (m, $20 \mathrm{H}), 0.87$ (s, 9H), 0.04 (s, 6H). To a suspension of methyltriphenylphosphonium bromide (13.08 g, $36.6 \mathrm{mmol})$ in THF $(150 \mathrm{~mL})$ at $0{ }^{\circ} \mathrm{C}$ was added NaHMDS (37 mL, 1.0 M in THF, 37 $\mathrm{mmol}$ ), stirred at $0{ }^{\circ} \mathrm{C}$ for $15 \mathrm{~min}$, warmed to room temperature, stirred for $1 \mathrm{~h}$, cooled to $0{ }^{\circ} \mathrm{C}$, and a solution of ketone 14, prepared in the previous step, in THF $(150 \mathrm{~mL})$ at $0{ }^{\circ} \mathrm{C}$ was added via cannula. The reaction mixture was stirred at $0{ }^{\circ} \mathrm{C}$ for $11 \mathrm{~h}$ and diluted with ether and water. The aqueous phase was extracted with ether $(3 \mathrm{x})$ and the combined organic extracts were washed with brine $(1 \mathrm{x})$, dried over $\mathrm{MgSO}_{4}$, and concentrated. Purification by flash column chromatography on silica gel (5\% to 10\% EtOAc in petroleum ether) gave alkene 24 (6.351 g, $60 \%)$ as a colorless oil: $\mathrm{R}_{f}=0.38\left(10 \%\right.$ EtOAc in petroleum ether); ${ }^{1} \mathrm{H} \mathrm{NMR}\left(400 \mathrm{MHz}, \mathrm{CDCl}_{3}\right)$ $\delta 7.26(\mathrm{~d}, J=8.2 \mathrm{~Hz}, 2 \mathrm{H}), 6.86(\mathrm{~d}, J=8.5 \mathrm{~Hz}, 2 \mathrm{H}), 5.34(\mathrm{~s}, 2 \mathrm{H}), 4.55-4.49(\mathrm{~m}, 2 \mathrm{H}), 4.25(\mathrm{~d}, J=$ $7.9 \mathrm{~Hz}, 1 \mathrm{H}), 4.11(\mathrm{~d}, J=8.1 \mathrm{~Hz}, 1 \mathrm{H}), 4.03(\mathrm{ddd}, J=9.0,6.4,2.3 \mathrm{~Hz}, 1 \mathrm{H}), 3.89$ (ddd, $J=8.9,8.9$, $2.6 \mathrm{~Hz}, 1 \mathrm{H}), 3.80(\mathrm{~s}, 3 \mathrm{H}), 3.78-3.66(\mathrm{~m}, 3 \mathrm{H}), 3.50(\mathrm{dd}, J=10.8,6.6 \mathrm{~Hz}, 1 \mathrm{H}), 1.97-1.56(\mathrm{~m}$, $18 \mathrm{H}), 0.88(\mathrm{~s}, 9 \mathrm{H}), 0.042(\mathrm{~s}, 3 \mathrm{H}), 0.038(\mathrm{~s}, 3 \mathrm{H}) ;{ }^{13} \mathrm{C} \mathrm{NMR}\left(101 \mathrm{MHz}, \mathrm{CDCl}_{3}\right) \delta 159.2,143.4$, 130.2, 129.3, 119.4, 118.6, 116.5, 113.7, 80.9, 80.6, 77.6, 77.2, 73.1, 69.4, 60.1, 55.2, 37.5, 37.4, 37.1 (2 carbons), 35.3, 25.9, 23.6, 23.4, 23.3 (2 carbons), 18.3, -5.3, -5.4; IR (neat) 2949, 1614, 1510, 1334, $1103 \mathrm{~cm}^{-1}$; MALDIMS $m / z 611.3[\mathrm{M}+\mathrm{Na}]^{+}$; optical rotation $[\alpha]^{25}{ }_{\mathrm{D}}-5.9^{\circ}(c 1.0$, $\mathrm{CH}_{2} \mathrm{Cl}_{2}$ ). Anal. Calcd for $\mathrm{C}_{33} \mathrm{H}_{52} \mathrm{O}_{7} \mathrm{Si}$ : C, 67.31; H, 8.90. Found: C, 67.15; H, 8.70.
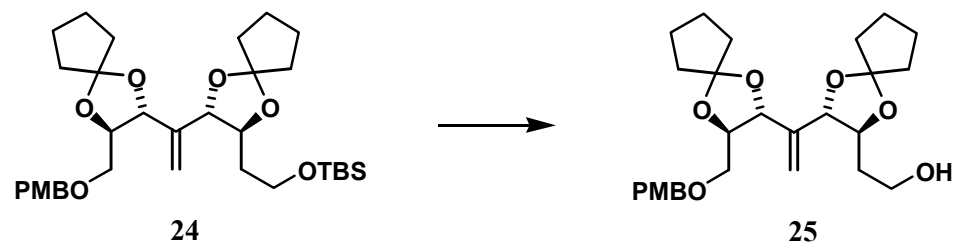

To a solution of ether $24(7.987 \mathrm{~g}, 13.6 \mathrm{mmol})$ in THF $(90 \mathrm{~mL})$ at room temperature was added TBAF (20.4 mL, $1 \mathrm{M}$ in THF, $20.4 \mathrm{mmol}$ ). The reaction mixture was stirred for $24 \mathrm{~h}$, and diluted with ether and water. The aqueous phase was extracted with ether $(4 \mathrm{x})$ and the combined organic extracts were washed with brine $(1 \mathrm{x})$, dried over $\mathrm{MgSO}_{4}$, and concentrated. Purification by flash column chromatography on silica gel (30\% to 50\% EtOAc in petroleum ether) gave alcohol 25 (5.024 g, 78\%) as a colorless oil: $\mathrm{R}_{f}=0.38$ (50\% EtOAc in petroleum ether); ${ }^{1} \mathrm{H}$ NMR $\left(400 \mathrm{MHz}, \mathrm{CDCl}_{3}\right) \delta 7.25(\mathrm{~d}, J=8.7 \mathrm{~Hz}, 2 \mathrm{H}), 6.86(\mathrm{~d}, J=8.7 \mathrm{~Hz}, 2 \mathrm{H}), 5.38(\mathrm{~s}, 1 \mathrm{H}), 5.36(\mathrm{~s}$, $1 \mathrm{H}), 4.51(\mathrm{~s}, 2 \mathrm{H}), 4.27$ (d, $J=8.1 \mathrm{~Hz}, 1 \mathrm{H}), 4.15(\mathrm{~d}, J=8.2 \mathrm{~Hz}, 1 \mathrm{H}), 4.02(\mathrm{ddd}, J=8.2,6.3,2.7$ 
$\mathrm{Hz}, 1 \mathrm{H}), 3.91$ (ddd, $J=8.4,8.4,3.4 \mathrm{~Hz}, 1 \mathrm{H}), 3.79$ (s, 3H), 3.78-3.75 (m, 2H), 3.66 (dd, $J=10.7$, $2.7 \mathrm{~Hz}, 1 \mathrm{H}), 3.50(\mathrm{dd}, J=10.8,6.3 \mathrm{~Hz}, 1 \mathrm{H}), 2.44(\mathrm{t}, J=5.6 \mathrm{~Hz}, 2 \mathrm{H}), 1.99-1.59(\mathrm{~m}, 17 \mathrm{H}) ;{ }^{13} \mathrm{C}$ NMR (101 MHz, $\left.\mathrm{CDCl}_{3}\right) \delta 159.1,143.0,130.1,129.35,129.30,119.5,118.9,117.2,113.7,80.9$, 80.32, 80.30, 77.2, 73.1, 69.1, 60.8, 55.2, 37.5, 37.4, 37.1, 34.0, 23.5, 23.4, 23.2 (2 carbons); IR (neat) 3486 (br), 2951, 1611, 1516, $1108 \mathrm{~cm}^{-1}$; MALDIMS m/z $497.3[\mathrm{M}+\mathrm{Na}]^{+}$; optical rotation $[\alpha]^{26}+4.4^{\circ}\left(c\right.$ 1.0, $\mathrm{CH}_{2} \mathrm{Cl}_{2}$ ); Anal. Calcd for $\mathrm{C}_{27} \mathrm{H}_{38} \mathrm{O}_{7}$ : C, 68.33; H, 8.07. Found: C, 68.17; H, 7.90 .
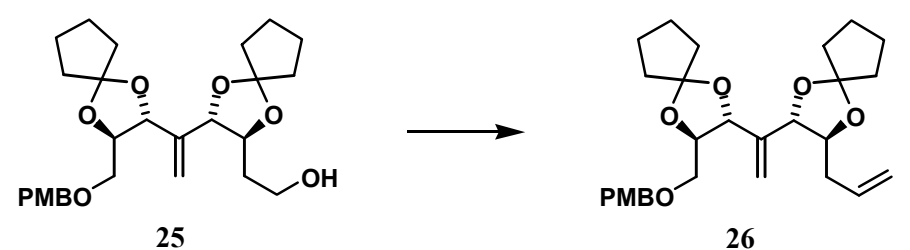

26

To a solution of oxalyl chloride $(1.50 \mathrm{~mL}, 2.18 \mathrm{~g}, 17.2 \mathrm{mmol})$ in $\mathrm{CH}_{2} \mathrm{Cl}_{2}(50 \mathrm{~mL})$ at -78 ${ }^{\circ} \mathrm{C}$ was added DMSO (2.45 mL, $\left.2.70 \mathrm{~g}, 34.5 \mathrm{mmol}\right)$, stirred for $15 \mathrm{~min}$, and a solution of alcohol 25 (5.083 g, $10.7 \mathrm{mmol})$ in $\mathrm{CH}_{2} \mathrm{Cl}_{2}(50 \mathrm{~mL})$ at $-78^{\circ} \mathrm{C}$ was added via cannula. After $1 \mathrm{~h}$ at -78 ${ }^{\circ} \mathrm{C}$, triethylamine $(7.5 \mathrm{~mL}, 5.4 \mathrm{~g}, 54 \mathrm{mmol})$ was added, the reaction mixture was stirred at $-78^{\circ} \mathrm{C}$ for 15 min, warmed to room temperature, diluted with $\mathrm{CH}_{2} \mathrm{Cl}_{2}$, washed with saturated $\mathrm{NH}_{4} \mathrm{Cl}$ (2 $\mathrm{x})$, brine (1 x), dried over $\mathrm{MgSO}_{4}$, filtered through a pad of silica gel, and concentrated to give the aldehyde which was used in the next step without further purification: $\mathrm{R}_{f}=0.32(30 \%$ EtOAc in petroleum ether); ${ }^{1} \mathrm{H}$ NMR $\left(500 \mathrm{MHz}, \mathrm{CDCl}_{3}\right) \delta 9.78(\mathrm{dd}, J=2.7,1.6 \mathrm{~Hz}, 1 \mathrm{H}), 7.26(\mathrm{~d}, J=8.7$ $\mathrm{Hz}, 2 \mathrm{H}), 6.87(\mathrm{~d}, J=8.8 \mathrm{~Hz}, 2 \mathrm{H}), 5.43(\mathrm{~s}, 1 \mathrm{H}), 5.32(\mathrm{~s}, 1 \mathrm{H}), 4.54-4.49(\mathrm{~m}, 2 \mathrm{H}), 4.30$ (d, $J=8.2$ $\mathrm{Hz}, 1 \mathrm{H}), 4.20$ (ddd, $J=8.2,8.2,3.2 \mathrm{~Hz}, 1 \mathrm{H}), 4.16$ (d, $J=8.1 \mathrm{~Hz}, 1 \mathrm{H}), 3.98$ (ddd, $J=8.6,6.0,2.7$ Hz, 1H), 3.80 (s, 3H), 3.64 (dd, $J=10.7,2.7 \mathrm{~Hz}, 1 \mathrm{H}), 3.50$ (dd, $J=10.8,6.0 \mathrm{~Hz}, 1 \mathrm{H}), 2.86$ (ddd, $J=16.6,3.1,1.5 \mathrm{~Hz}, 1 \mathrm{H}), 2.61(\mathrm{ddd}, J=16.6,8.2,2.6 \mathrm{~Hz}, 1 \mathrm{H}), 1.92-1.61$ (m, 16H). To a suspension of methyltriphenylphosphonium bromide $(6.00 \mathrm{~g}, 16.8 \mathrm{mmol})$ in THF $(65 \mathrm{~mL})$ at 0 ${ }^{\circ} \mathrm{C}$ was added $n$-BuLi $(6.70 \mathrm{~mL}, 2.5 \mathrm{M}$ in hexanes, $16.8 \mathrm{mmol})$. The solution was stirred at $0{ }^{\circ} \mathrm{C}$ for $30 \mathrm{~min}$ and a solution of the aldehyde, prepared in the previous step, in THF (65 mL) at room temperature was added via cannula. The reaction mixture was warmed to room temperature over $1 \mathrm{~h}$, stirred at room temperature for $14 \mathrm{~h}$, and diluted with ether and water. The aqueous phase was extracted with ether $(5 \mathrm{x})$ and the combined organic extracts were washed with brine $(1 \mathrm{x})$, dried over $\mathrm{MgSO}_{4}$, and concentrated. Purification by flash column chromatography on silica gel 
(5\% to $10 \%$ to $20 \%$ EtOAc in petroleum ether) gave alkene 26 (3.990 g, 79\%) as a colorless oil: $\mathrm{R}_{f}=0.55\left(20 \%\right.$ EtOAc in petroleum ether); ${ }^{1} \mathrm{H}$ NMR $\left(400 \mathrm{MHz}, \mathrm{CDCl}_{3}\right) \delta 7.26(\mathrm{~d}, J=8.2 \mathrm{~Hz}$, $2 \mathrm{H}), 6.86(\mathrm{~d}, J=8.7 \mathrm{~Hz}, 2 \mathrm{H}), 5.86(\mathrm{dddd}, \mathrm{J}=17.1,13.6,10.2,6.9 \mathrm{~Hz}, 1 \mathrm{H}), 5.36(\mathrm{~s}, 1 \mathrm{H}), 5.34(\mathrm{~s}$, $1 \mathrm{H}), 5.11(\mathrm{dm}, \mathrm{J}=17.2 \mathrm{~Hz}, 1 \mathrm{H}), 5.08(\mathrm{dm}, \mathrm{J}=10.2 \mathrm{~Hz}, 1 \mathrm{H}), 4.55-4.49(\mathrm{~m}, 2 \mathrm{H}), 4.27(\mathrm{~d}, \mathrm{~J}=7.9$ $\mathrm{Hz}, 1 \mathrm{H}$ ), 4.15 (d, J = 7.9 Hz, 1H), 4.02 (ddd, J = 8.1, 6.4, 2.6 Hz, 1H), 3.85 (ddd, J = 7.9, 7.9, 3.8 Hz, 1H), 3.80 (s, 3H), 3.69 (dd, J = 10.7, $2.8 \mathrm{~Hz}, 1 \mathrm{H}), 3.50$ (dd, J = 10.7, $6.6 \mathrm{~Hz}, 1 \mathrm{H}), 2.49$ (m, $1 \mathrm{H}), 2.26(\mathrm{ddd}, \mathrm{J}=14.8,7.5,7.5 \mathrm{~Hz}, 1 \mathrm{H}), 1.94-1.56(\mathrm{~m}, 16 \mathrm{H}) ;{ }^{13} \mathrm{C}$ NMR $\left(101 \mathrm{MHz}, \mathrm{CDCl}_{3}\right) \delta$ 159.4, 143.7, 134.5, 130.4, 129.6, 119.7, 119.0, 117.5, 116.9, 113.9, 80.9, 80.7, 80.3, 77.4, 73.3, 69.6, 55.5, 37.8, 37.7, 37.41, 37.38, 36.5, 23.8, 23.7, 23.6 (2 carbons); IR (neat) 2959, 1614, 1515, $1103 \mathrm{~cm}^{-1}$; EIMS m/z 137 (2), 123 (2), 122 (4), 121 (100); HREIMS calcd for $\mathrm{C}_{28} \mathrm{H}_{38} \mathrm{O}_{6}$ 470.2663 , found 470.2668 ; optical rotation $[\alpha]^{24}{ }_{\mathrm{D}}+0.5^{\circ}\left(c 1.0, \mathrm{CH}_{2} \mathrm{Cl}_{2}\right)$. Anal. Calcd for $\mathrm{C}_{28} \mathrm{H}_{38} \mathrm{O}_{6}: \mathrm{C}, 71.46 ; \mathrm{H}, 8.14$. Found: C, 71.65; H, 7.98.
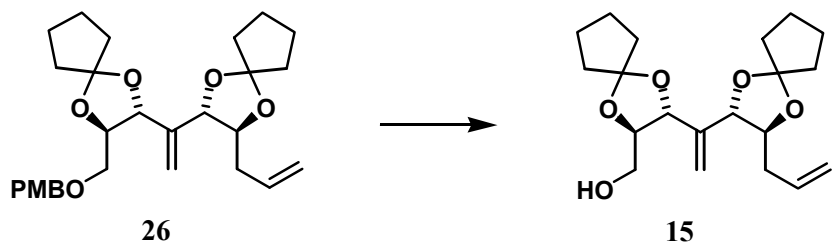

To a solution of ether $26(3.990 \mathrm{~g}, 8.48 \mathrm{mmol})$ in $\mathrm{CH}_{2} \mathrm{Cl}_{2}(55 \mathrm{~mL})$ and water $(20 \mathrm{~mL})$ at room temperature was added DDQ $(2.359 \mathrm{~g}, 10.4 \mathrm{mmol})$. The reaction mixture was stirred at room temperature for $2 \mathrm{~h}$ and diluted with ether, water, and saturated $\mathrm{NaHCO}_{3}$. The aqueous phase was extracted with ether $(4 \mathrm{x})$ and the combined organic extracts were washed with brine $(1 \mathrm{x})$, dried over $\mathrm{MgSO}_{4}$, and concentrated. Purification by flash column chromatography on silica gel ( $0 \%$ to $50 \%$ EtOAc in $\left.\mathrm{CH}_{2} \mathrm{Cl}_{2}\right)$ gave alkene $15(2.918 \mathrm{~g}, 98 \%)$ as a colorless oil: $\mathrm{R}_{f}=$ 0.32 (30\% EtOAc in petroleum ether); ${ }^{1} \mathrm{H}$ NMR ( $\left.500 \mathrm{MHz}, \mathrm{CDCl}_{3}\right) \delta 5.86$ (dddd, $J=17.1,13.7$, $10.3,7.0 \mathrm{~Hz}, 1 \mathrm{H}), 5.51(\mathrm{~s}, 1 \mathrm{H}), 5.36$ (s, $1 \mathrm{H}), 5.15-5.09$ (m, 2H), 4.27 (d, J= 7.2 Hz, 1H), 4.19 (d, $J=8.2 \mathrm{~Hz}, 1 \mathrm{H}), 3.86$ (ddd, $J=7.7,7.7,3.7 \mathrm{~Hz}, 1 \mathrm{H}), 3.83-3.75$ (m, 3H), $2.68(\mathrm{~s} \mathrm{br}, 1 \mathrm{H}), 2.48$ $(\mathrm{dm}, J=14.8 \mathrm{~Hz}, 1 \mathrm{H}), 2.28$ (ddd, $J=14.8,7.3,7.3 \mathrm{~Hz}, 1 \mathrm{H}), 1.90-1.61(\mathrm{~m}, 16 \mathrm{H}) ;{ }^{13} \mathrm{C}$ NMR $(126$ $\left.\mathrm{MHz}, \mathrm{CDCl}_{3}\right) \delta 142.7,133.8,119.1,118.7,117.60,117.59,83.2,81.8,79.3,77.1,62.0,37.4(2$ carbons), 37.3, 37.1, 36.0, 23.43, 23.39, 23.37, 23.2; IR (neat) 3484 (br), 2959, 1642, $1107 \mathrm{~cm}^{-1}$; EIMS m/z 277350 (13), 322 (17), 321 (37), 225 (15); HREIMS calcd for $\mathrm{C}_{20} \mathrm{H}_{30} \mathrm{O}_{5}$ 350.2093, 
found 350.2090; optical rotation $[\alpha]^{24}-22^{\circ}\left(c 1.0, \mathrm{CH}_{2} \mathrm{Cl}_{2}\right)$. Anal. Calcd for $\mathrm{C}_{20} \mathrm{H}_{30} \mathrm{O}_{5}$ : C, 68.54; H, 8.63. Found: C, 68.70; H, 8.66.

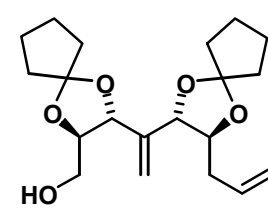

15

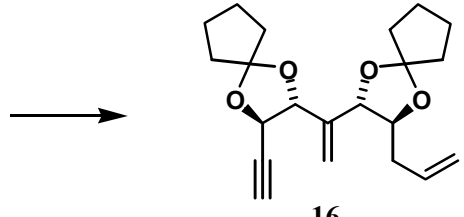

16

To a solution of oxalyl chloride ( $40 \mu \mathrm{L}, 58 \mathrm{mg}, 0.46 \mathrm{mmol})$ in $\mathrm{CH}_{2} \mathrm{Cl}_{2}(2 \mathrm{~mL})$ at $-78{ }^{\circ} \mathrm{C}$ was added DMSO ( $65 \mu \mathrm{L}, 72 \mathrm{mg}, 0.92 \mathrm{mmol}$ ), stirred for $15 \mathrm{~min}$, and a solution of alcohol 15 (101 mg, $0.29 \mathrm{mmol})$ in $\mathrm{CH}_{2} \mathrm{Cl}_{2}(2 \mathrm{~mL})$ at $-78^{\circ} \mathrm{C}$ was added via cannula. After $1 \mathrm{~h}$ at $-78^{\circ} \mathrm{C}$, triethylamine $(200 \mu \mathrm{L}, 145 \mathrm{mg}, 1.4 \mathrm{mmol})$ was added, the reaction mixture was stirred at $-78^{\circ} \mathrm{C}$ for $15 \mathrm{~min}$, warmed to $0{ }^{\circ} \mathrm{C}$, diluted with ether, washed with saturated $\mathrm{NH}_{4} \mathrm{Cl}(2 \mathrm{x})$, brine $(1 \mathrm{x})$, dried over $\mathrm{MgSO}_{4}$, filtered through a pad of silica gel, and concentrated to give the aldehyde which was used in the next step without further purification: $\mathrm{R}_{f}=0.19$ (20\% EtOAc in petroleum ether); ${ }^{1} \mathrm{H}$ NMR (400 MHz, $\mathrm{CDCl}_{3}$ ) $\delta 9.72(\mathrm{~d}, \mathrm{~J}=2.0 \mathrm{~Hz}, 1 \mathrm{H}$ ), 5.87 (dddd, $J=16.9,13.6,10.2$, $6.9 \mathrm{~Hz}, 1 \mathrm{H}), 5.51(\mathrm{~s}, 1 \mathrm{H}), 5.32(\mathrm{~s}, 1 \mathrm{H}), 5.16-5.10(\mathrm{~m}, 2 \mathrm{H}), 4.66(\mathrm{~d}, J=6.6 \mathrm{~Hz}, 1 \mathrm{H}), 4.23(\mathrm{dd}, J=$ 6.7, $2.0 \mathrm{~Hz}, 1 \mathrm{H}), 4.20$ (d, $J=8.2 \mathrm{~Hz}, 1 \mathrm{H}), 3.86$ (ddd, $J=7.6,7.6,3.8 \mathrm{~Hz}, 1 \mathrm{H}), 2.46$ (dm, $J=14.8$ $\mathrm{Hz}, 1 \mathrm{H}), 2.28$ (ddd, $J=14.6,6.9,6.9 \mathrm{~Hz}, 1 \mathrm{H}), 2.00-1.62(\mathrm{~m}, 16 \mathrm{H})$. To a solution of $(\mathrm{MeO})_{2} \mathrm{POC}\left(=\mathrm{N}_{2}\right) \mathrm{COMe}(277 \mathrm{mg}, 1.44 \mathrm{mmol})$ in $\mathrm{MeOH}(1 \mathrm{~mL})$ at $0{ }^{\circ} \mathrm{C}$ was added $\mathrm{K}_{2} \mathrm{CO}_{3}(204$ $\mathrm{mg}, 1.48 \mathrm{mmol})$. After $30 \mathrm{~min}$, a solution of the aldehyde, prepared in the previous step, in $\mathrm{MeOH}(1.5 \mathrm{~mL})$ at $0{ }^{\circ} \mathrm{C}$ was added via cannula. The reaction mixture was warmed to room temperature over $1 \mathrm{~h}$, stirred at room temperature for $24 \mathrm{~h}$, and diluted with ether and water. The aqueous phase was extracted with ether $(4 \mathrm{x})$ and the combined organic extracts were washed with brine (1 x), dried over $\mathrm{MgSO}_{4}$, and concentrated. Purification by flash column chromatography on silica gel (5\% to 10\% EtOAc in petroleum ether) gave alkyne $\mathbf{1 6}$ (86 mg, $87 \%)$ as a colorless oil: $\mathrm{R}_{f}=0.45\left(10 \%\right.$ EtOAc in petroleum ether); ${ }^{1} \mathrm{H} \mathrm{NMR}\left(500 \mathrm{MHz}, \mathrm{CDCl}_{3}\right)$ $\delta 5.87$ (dddd, $J=17.1,13.7,10.1,6.8 \mathrm{~Hz}, 1 \mathrm{H}), 5.44(\mathrm{~s}, 1 \mathrm{H}), 5.33(\mathrm{~s}, 1 \mathrm{H}), 5.15-5.08(\mathrm{~m}, 2 \mathrm{H})$, $4.58(\mathrm{~d}, J=6.1 \mathrm{~Hz}, 1 \mathrm{H}), 4.52$ (dd, $J=6.2,2.2 \mathrm{~Hz}, 1 \mathrm{H}), 4.17$ (d, $J=7.9 \mathrm{~Hz}, 1 \mathrm{H}), 3.89$ (ddd, $J=$ 7.7, 7.7, $3.8 \mathrm{~Hz}, 1 \mathrm{H}$ ), 2.50 (d, $J=2.1 \mathrm{~Hz}, 1 \mathrm{H}$ ), 2.47 (m, 1H), 2.29 (ddddd, $J=14.7,7.3,7.3,1.3$, $1.3 \mathrm{~Hz}, 1 \mathrm{H}), 2.06-1.61(\mathrm{~m}, 16 \mathrm{H}){ }^{13} \mathrm{C}$ NMR $\left(126 \mathrm{MHz}, \mathrm{CDCl}_{3}\right) \delta 142.9,134.1,120.7,119.1$, $117.4,116.1,81.7,81.2,80.4,79.5,74.4,70.6,37.6,37.1,36.7,36.6,36.3,23.9,23.5,23.4$, 
22.9; IR (neat) 3303, 2959, 1642, 1334, 1207, $1107 \mathrm{~cm}^{-1}$; ESIMS $m / z 367.4[\mathrm{M}+\mathrm{Na}]^{+}$; optical rotation $[\alpha]^{24}+1.6^{\circ}\left(c 1.0, \mathrm{CH}_{2} \mathrm{Cl}_{2}\right)$; Anal. Calcd for $\mathrm{C}_{21} \mathrm{H}_{28} \mathrm{O}_{4}$ : $\mathrm{C}, 73.23 ; \mathrm{H}, 8.19$. Found: $\mathrm{C}$, $73.35 ; \mathrm{H}, 8.28$.

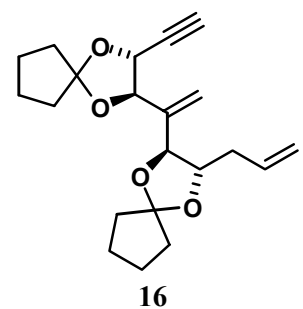

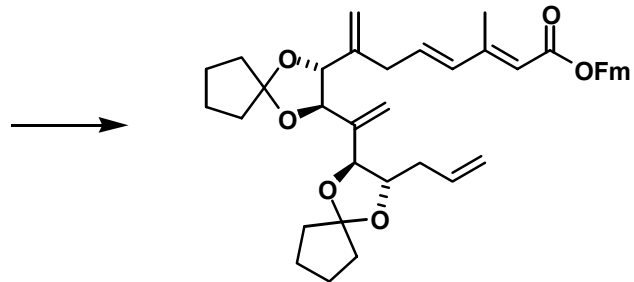

18

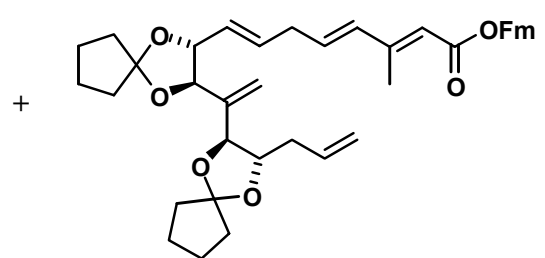

27

A solution of alkyne $16(576 \mathrm{mg}, 1.67 \mathrm{mmol})$ and alkene $17(2.581 \mathrm{~g}, 8.48 \mathrm{mmol})$ in DCE $(4.3 \mathrm{~mL})$ was degassed by bubbling argon through the solution for $15 \mathrm{~min}$, the reaction mixture was heated to $50^{\circ} \mathrm{C}$, and $\mathrm{Cp} * \mathrm{Ru}(\mathrm{MeCN}){ }_{3} \mathrm{PF}_{6}(87 \mathrm{mg}, 0.17 \mathrm{mmol})$ was added in one portion. After $1 \mathrm{~h}$, the reaction mixture was filtered through silica gel and concentrated. Purification by flash column chromatography on silica gel (3\% to $6 \%$ to $9 \%$ EtOAc in petroleum ether) gave branched alkene 18 (251 mg, 23\%), linear alkene 27 (94 mg, 9\%) and recovered alkyne 16 (233 mg, 40\%) as colorless oils. Data for 18: $\mathrm{R}_{f}=0.30(10 \%$ EtOAc in petroleum ether); ${ }^{1} \mathrm{H}$ NMR (500 MHz, $\left.\mathrm{CDCl}_{3}\right) \delta 7.77(\mathrm{~d}, J=7.6 \mathrm{~Hz}, 2 \mathrm{H}), 7.62(\mathrm{~d}, J=7.5 \mathrm{~Hz}, 2 \mathrm{H}), 7.41$ (dd, $J=7.5,7.5 \mathrm{~Hz}, 2 \mathrm{H}), 7.32(\mathrm{dd}, J=7.4,7.4 \mathrm{~Hz}, 2 \mathrm{H}), 6.22-6.14(\mathrm{~m}, 2 \mathrm{H}), 5.89$ (dddd, $J=17.1$, 13.7, 10.1, 6.7 Hz, 1H), $5.83(\mathrm{~s}, 1 \mathrm{H}), 5.43(\mathrm{~s}, 1 \mathrm{H}), 5.38(\mathrm{~s}, 1 \mathrm{H}), 5.25(\mathrm{~s}, 1 \mathrm{H}), 5.16-5.10(\mathrm{~m}, 2 \mathrm{H})$, $5.00(\mathrm{~s}, 1 \mathrm{H}), 4.42(\mathrm{~d}, J=7.2 \mathrm{~Hz}, 2 \mathrm{H}), 4.32-4.24(\mathrm{~m}, 3 \mathrm{H}), 4.18$ (d, $J=7.8 \mathrm{~Hz}, 1 \mathrm{H}), 3.93$ (ddd, $J=$ 7.7, 7.7, 3.8 Hz, 1H), $3.03(\mathrm{dd}, J=16.6,5.9 \mathrm{~Hz}, 1 \mathrm{H}), 2.92(\mathrm{dd}, J=16.6,6.7 \mathrm{~Hz}, 1 \mathrm{H}), 2.52(\mathrm{~m}$, 1H), 2.31 (ddd, $J=14.5,7.5,7.5 \mathrm{~Hz}, 1 \mathrm{H}), 2.27$ (s, 3H), 1.93-1.62 (m, 16H); ${ }^{13} \mathrm{C}$ NMR $(126$ $\left.\mathrm{MHz}, \mathrm{CDCl}_{3}\right) \delta 167.0,153.0,144.0,143.3,142.8,141.3,135.5,134.4,134.3,127.7,127.0$, $125.1,119.9,119.0,118.8,117.8,117.6,117.2$, 114.7, 83.0, 80.3, 80.2, 79.4, 65.7, 46.8, 37.6, 37.4, 37.30, 37.25, 36.3, 35.1, 23.5, 23.4 (2 carbons), 23.3, 13.9; IR (neat) 2959, 1715, 1613, 1147, $1107 \mathrm{~cm}^{-1}$; EIMS m/z 180 (21), 165 (20); HREIMS calcd for $\mathrm{C}_{42} \mathrm{H}_{48} \mathrm{O}_{6} 648.3451$, found 648.3456; optical rotation $[\alpha]^{24}+21^{\circ}\left(c 0.50, \mathrm{CH}_{2} \mathrm{Cl}_{2}\right)$. Data for 27: $\mathrm{R}_{f}=0.26(10 \% \mathrm{EtOAc}$ in petroleum ether); ${ }^{1} \mathrm{H}$ NMR $\left(500 \mathrm{MHz}, \mathrm{CDCl}_{3}\right) \delta 7.77(\mathrm{~d}, J=7.5 \mathrm{~Hz}, 2 \mathrm{H}), 7.62(\mathrm{~d}, J=7.5 \mathrm{~Hz}$, 2H), $7.41(\mathrm{t}, J=7.5 \mathrm{~Hz}, 2 \mathrm{H}), 7.32(\mathrm{t}, J=7.5 \mathrm{~Hz}, 2 \mathrm{H}), 6.19-6.13(\mathrm{~m}, 2 \mathrm{H}), 5.93-5.80(\mathrm{~m}, 2 \mathrm{H}), 5.83$ $(\mathrm{s}, 1 \mathrm{H}), 5.56(\mathrm{dd}, J=15.4,6.8 \mathrm{~Hz}, 1 \mathrm{H}), 5.48-5.09(\mathrm{~m}, 3 \mathrm{H}), 5.38(\mathrm{~s}, 1 \mathrm{H}), 4.42(\mathrm{~d}, J=7.2 \mathrm{~Hz}, 1 \mathrm{H})$, 4.30-4.06 (m, 5H), $3.91(\mathrm{~m}, 1 \mathrm{H}), 2.95(\mathrm{dd}, J=5.9,5.9 \mathrm{~Hz}, 1 \mathrm{H}), 2.52(\mathrm{dm}, J=10.4 \mathrm{~Hz}, 1 \mathrm{H})$, 
2.33-2.27 (m, 2H), 2.25 (s, 3H), 1.89-1.65 (m, 16H); $\left.{ }^{13} \mathrm{C} \mathrm{NMR} \mathrm{(126} \mathrm{MHz,} \mathrm{CDCl}_{3}\right) \delta$ 166.9, $152.9,144.0,142.7,141.2,134.7,134.3,134.2,132.3,128.2,127.7,127.0,125.1,119.9,118.9$, $118.8,117.9,117.2,116.5,81.9,80.9,80.01,79.96,65.7,46.8,37.6,37.5,37.3,37.2,36.4,35.5$, $23.50,23.47,23.4,23.3,13.8$.

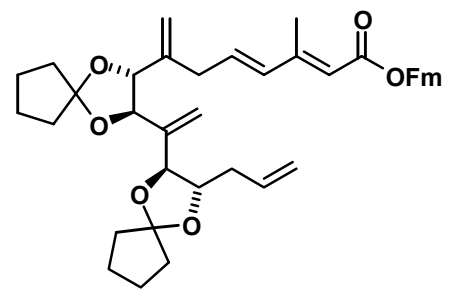

18

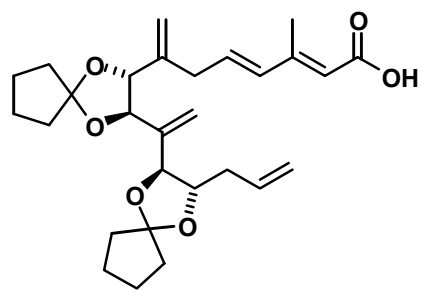

28

To a solution of ester $18(310 \mathrm{mg}, 0.48 \mathrm{mmol})$ in $\mathrm{CH}_{2} \mathrm{Cl}_{2}(14 \mathrm{~mL})$ at room temperature was added piperidine $(1.6 \mathrm{~mL}, 1.4 \mathrm{~g}, 16 \mathrm{mmol})$. After $3 \mathrm{~h}$ at room temperature, the reaction mixture was diluted with ether, water, and $1 \mathrm{M} \mathrm{HCl}$. The aqueous phase was extracted with ether $(3 \mathrm{x})$ and the combined organic extracts were washed with brine $(1 \mathrm{x})$, dried over $\mathrm{MgSO}_{4}$, and concentrated. Purification by flash column chromatography on silica gel (30\% to $50 \%$ EtOAc in petroleum ether) gave acid $28(198 \mathrm{mg}, 88 \%)$ as a colorless oil: $\mathrm{R}_{f}=0.41(40 \% \mathrm{EtOAc}$ in petroleum ether); ${ }^{1} \mathrm{H}$ NMR $\left(500 \mathrm{MHz}, \mathrm{CDCl}_{3}\right) \delta$ 6.18-6.14 (m, 2H), 5.88 (dddd, $J=17.1,13.7$, 10.3, $6.7 \mathrm{~Hz}, 1 \mathrm{H}), 5.74(\mathrm{~d}, J=0.9 \mathrm{~Hz}, 1 \mathrm{H}), 5.41(\mathrm{~s}, 1 \mathrm{H}), 5.36(\mathrm{~s}, 1 \mathrm{H}), 5.23(\mathrm{~s}, 1 \mathrm{H}), 5.15-5.08$ (m, 2H), $4.97(\mathrm{~d}, J=1.1 \mathrm{~Hz}, 1 \mathrm{H}), 4.29(\mathrm{~d}, J=8.4 \mathrm{~Hz}, 1 \mathrm{H}), 4.26(\mathrm{~d}, J=8.4 \mathrm{~Hz}, 1 \mathrm{H}), 4.16(\mathrm{~d}, J=7.7$ $\mathrm{Hz}, 1 \mathrm{H}), 3.91$ (ddd, $J=7.7,7.7,3.8 \mathrm{~Hz}, 1 \mathrm{H}), 3.02$ (dd, $J=16.2,4.5 \mathrm{~Hz}, 1 \mathrm{H}), 2.91$ (dd, $J=16.1$, $5.3 \mathrm{~Hz}, 1 \mathrm{H}), 2.51(\mathrm{dm}, J=14.7 \mathrm{~Hz}, 1 \mathrm{H}), 2.31(\mathrm{~m}, 1 \mathrm{H}), 2.29$ (d, $J=1.0 \mathrm{~Hz}, 3 \mathrm{H}), 1.90-1.62(\mathrm{~m}$, $17 \mathrm{H}) ;{ }^{13} \mathrm{C}$ NMR $\left(126 \mathrm{MHz}, \mathrm{CDCl}_{3}\right) \delta 172.0,154.7,143.2,142.8,135.5,135.1,134.3,119.0$, 118.8, 117.6, 117.4, 117.3, 114.8, 83.1, 80.3, 80.2, 79.4, 37.6, 37.4, 37.30, 37.25, 36.3, 35.1, 23.5, 23.41, 23.40, 23.3, 14.1; IR (neat) 3076 (br), 2959, 1682, 1610, $1107 \mathrm{~cm}^{-1}$; MALDIMS m/z $493.2[\mathrm{M}+\mathrm{Na}]^{+}$; optical rotation $[\alpha]^{28}{ }_{\mathrm{D}}+28^{\circ}\left(c 0.50, \mathrm{CH}_{2} \mathrm{Cl}_{2}\right)$.
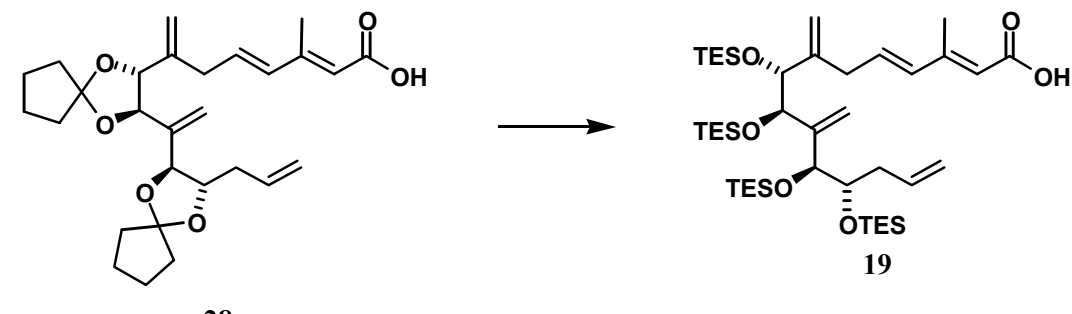
To ketal 28 (76 mg, $0.16 \mathrm{mmol})$ at room temperature was added acetic acid $(1.5 \mathrm{~mL})$ and water $(500 \mu \mathrm{L})$. The reaction mixture was heated to $40^{\circ} \mathrm{C}$ for $20 \mathrm{~h}$ and concentrated to give the tetraol which was used in the next step without further purification. To a solution of the tetraol, prepared in the previous step, in THF $(4 \mathrm{~mL})$ at $0{ }^{\circ} \mathrm{C}$ was added $i$ - $\operatorname{Pr}_{2} \mathrm{NEt}(260 \mu \mathrm{L}, 193 \mathrm{mg}, 1.5$ $\mathrm{mmol})$ and TESOTf $(230 \mu \mathrm{L}, 269 \mathrm{mg}, 1.0 \mathrm{mmol})$. The reaction mixture was stirred at $0{ }^{\circ} \mathrm{C}$ for 10 min, quenched with $1 \mathrm{M} \mathrm{HCl}$, stirred for $10 \mathrm{~min}$, and diluted with ether and water. The aqueous phase was extracted with ether $(3 \mathrm{x})$ and the combined organic extracts were washed with saturated $\mathrm{KH}_{2} \mathrm{PO}_{4}(1 \mathrm{x})$, brine $(1 \mathrm{x})$, dried over $\mathrm{MgSO}_{4}$, and concentrated. Purification by flash column chromatography on silica gel (5\% to 10\% EtOAc in petroleum ether) gave silyl ether 19 (106 mg, 83\%) as a colorless oil: $\mathrm{R}_{f}=0.56$ (30\% EtOAc in petroleum ether); ${ }^{1} \mathrm{H}$ NMR (500 $\left.\mathrm{MHz}, \mathrm{CDCl}_{3}\right) \delta 6.20(\mathrm{ddd}, J=15.6,6.8,6.8 \mathrm{~Hz}, 1 \mathrm{H}), 6.14(\mathrm{~d}, J=15.6 \mathrm{~Hz}, 1 \mathrm{H}), 5.82(\mathrm{dddd}, J=$ 17.0, 14.3, 10.3, 7.1 Hz, 1H), 5.40-5.39 (m, 2H), $5.09(\mathrm{~s}, 1 \mathrm{H}), 5.05(\mathrm{dd}, J=9.6,2.0 \mathrm{~Hz}, 1 \mathrm{H})$, $5.02(\mathrm{~d}, J=0.9 \mathrm{~Hz}, 1 \mathrm{H}), 4.79(\mathrm{~d}, J=1.3 \mathrm{~Hz}, 1 \mathrm{H}), 4.26(\mathrm{~d}, J=4.0 \mathrm{~Hz}, 1 \mathrm{H}), 4.23(\mathrm{~d}, J=3.8 \mathrm{~Hz}$, $1 \mathrm{H}), 4.21(\mathrm{~d}, J=3.7 \mathrm{~Hz}, 1 \mathrm{H}), 3.80(\mathrm{ddd}, J=7.9,3.9,3.9 \mathrm{~Hz}, 1 \mathrm{H}), 3.01(\mathrm{dd}, J=17.5,6.6 \mathrm{~Hz}$, 1H), $2.95(\mathrm{dd}, J=17.2,6.6 \mathrm{~Hz}, 1 \mathrm{H}), 2.40(\mathrm{dm}, J=14.2 \mathrm{~Hz}, 1 \mathrm{H}), 2.31(\mathrm{~d}, J=1.1 \mathrm{~Hz}, 3 \mathrm{H}), 2.30$ (m, 1H), 2.14 (ddd, $J=15.4,7.7,7.7 \mathrm{~Hz}, 1 \mathrm{H}), 0.99-0.91(\mathrm{~m}, 36 \mathrm{H}), 0.65-0.51(\mathrm{~m}, 24 \mathrm{H})$, exchangeable proton not listed; ${ }^{13} \mathrm{C} \mathrm{NMR}\left(126 \mathrm{MHz}, \mathrm{CDCl}_{3}\right) \delta 171.6,155.0,148.7,146.5,136.4$, 136.1, 135.0, 117.2, 116.9, 116.6, 112.9, 78.1, 75.9, 75.8, 75.0, 37.1, 35.4, 14.0, 7.1, 7.05, 7.04, $7.01,5.4,5.32,5.28,5.1$; IR (neat) $2956,1684,1610,1096,738 \mathrm{~cm}^{-1}$; optical rotation $[\alpha]^{23} \mathrm{D}^{-}$ $1.3^{\circ}$ (c $0.20, \mathrm{CH}_{2} \mathrm{Cl}_{2}$ ).

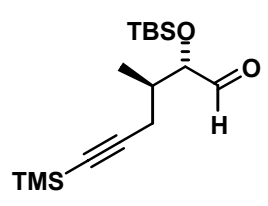

29

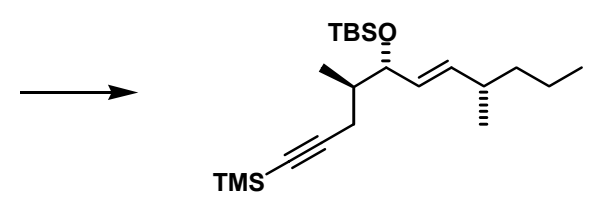

30

To $\mathrm{KN}(\mathrm{TMS})_{2}(6.4 \mathrm{~mL}, 0.47 \mathrm{M}$ in DME, $3.0 \mathrm{mmol})$ at $-78^{\circ} \mathrm{C}$ was added a solution of 5((S)-2-methyl-butane-1-sulfonyl)-1-phenyl-1H-tetrazole $(823 \mathrm{mg}, 2.80 \mathrm{mmol})$ in DME (6 mL) at $-78^{\circ} \mathrm{C}$. The solution was stirred at $-78^{\circ} \mathrm{C}$ for $1 \mathrm{~h}$ and a solution of aldehyde $29(677 \mathrm{mg}, 2.17$ $\mathrm{mmol})$ in $\mathrm{DME}(6 \mathrm{~mL})$ at $-78^{\circ} \mathrm{C}$ was added via cannula. The reaction mixture was warmed from $78^{\circ} \mathrm{C}$ to room temperature over $3 \mathrm{~h}$, stirred at room temperature for $10 \mathrm{~h}$, partially concentrated, and diluted with ether and water. The aqueous phase was extracted with ether $(5 \mathrm{x})$ and the combined organic extracts were washed with brine $(1 \mathrm{x})$, dried over $\mathrm{MgSO}_{4}$, and concentrated. 
Purification by flash column chromatography on silica gel (1\% to $2 \%$ EtOAc in petroleum ether) gave alkene 30 (614 mg, 74\%) as a colorless oil: $\mathrm{R}_{f}=0.71$ (5\% EtOAc in petroleum ether); ${ }^{1} \mathrm{H}$ NMR (400 MHz, $\left.\mathrm{CDCl}_{3}\right) \delta 5.39(\mathrm{dd}, J=15.4,7.3 \mathrm{~Hz}, 1 \mathrm{H}), 5.30(\mathrm{dd}, J=15.4,6.9 \mathrm{~Hz}, 1 \mathrm{H}), 4.04$ (dd, $J=6.9,4.9 \mathrm{~Hz}, 1 \mathrm{H}), 2.30$ (dd, $J=16.8,5.8 \mathrm{~Hz}, 1 \mathrm{H}), 2.10$ (m, 1H), 2.05 (dd, $J=16.9,7.8$ $\mathrm{Hz}, 1 \mathrm{H}), 1.68(\mathrm{~m}, 1 \mathrm{H}), 1.32-1.21(\mathrm{~m}, 4 \mathrm{H}), 0.963(\mathrm{~d}, J=6.7 \mathrm{~Hz}, 3 \mathrm{H}), 0.957$ (d, $J=6.7 \mathrm{~Hz}, 3 \mathrm{H})$, 0.89-0.86 (m, 3H), 0.88 (s, 9H), 0.15 (s, 9H), 0.04 (s, 3H), 0.00 (s, 3H); ${ }^{13} \mathrm{C}$ NMR (101 MHz, $\left.\mathrm{CDCl}_{3}\right) \delta 137.7,129.8,106.9,85.3,76.0,39.6,39.2,36.3,25.9,23.7,20.6,20.5,18.2,14.4,14.1$, 0.17, -4.0, -4.9; IR (neat) 2953, 2176, 1247, 1032, $842 \mathrm{~cm}^{-1}$; EIMS m/z 147 (57), 81 (50), 73 (97), 71 (69), 69 (100); HREIMS calcd for $\mathrm{C}_{18} \mathrm{H}_{35} \mathrm{OSi}_{2}\left(\mathrm{M}^{+}\right.$- tert-butyl) 323.2226, found 323.2191; optical rotation $[\alpha]^{26}+24^{\circ}\left(c 1.0, \mathrm{CH}_{2} \mathrm{Cl}_{2}\right)$.

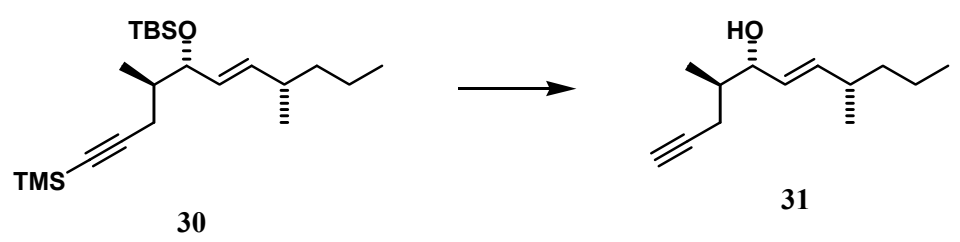

To a solution of silyl ether $\mathbf{3 0}(608 \mathrm{mg}, 1.60 \mathrm{mmol})$ in THF $(3.5 \mathrm{~mL})$ at room temperature was added TBAF ( $3.5 \mathrm{~mL}, 1 \mathrm{M}$ in THF, $3.5 \mathrm{mmol}$ ). The reaction mixture was stirred at room temperature for $24 \mathrm{~h}$ and concentrated. Purification by flash column chromatography on silica gel (5\% to $10 \%$ to $20 \%$ EtOAc in petroleum ether) gave alcohol $31(272 \mathrm{mg}, 88 \%)$ as a colorless oil: $\mathrm{R}_{f}=0.41$ (20\% EtOAc in petroleum ether); ${ }^{1} \mathrm{H}$ NMR (400 MHz, $\left.\mathrm{CDCl}_{3}\right) \delta 5.53$ (ddd, $J=$ 15.4, 7.8, $0.9 \mathrm{~Hz}, 1 \mathrm{H}$ ), 5.40 (ddd, $J=15.4,7.0,0.9 \mathrm{~Hz}, 1 \mathrm{H}$ ), 4.06 (m, 1H), 2.32 (ddd, $J=16.8$, 5.8, $2.6 \mathrm{~Hz}, 1 \mathrm{H}), 2.13(\mathrm{~m}, 1 \mathrm{H}), 2.08$ (ddd, $J=16.8,7.6,2.8 \mathrm{~Hz}, 1 \mathrm{H}), 1.97$ (t, $J=2.6 \mathrm{~Hz}, 1 \mathrm{H})$, $1.80(\mathrm{~m}, 1 \mathrm{H}), 1.60(\mathrm{~d} \mathrm{br}, J=3.8 \mathrm{~Hz}, 1 \mathrm{H}), 1.30-1.23(\mathrm{~m}, 4 \mathrm{H}), 1.02(\mathrm{~d}, J=6.9 \mathrm{~Hz}, 3 \mathrm{H}), 0.97$ (d, $J$ $=6.7 \mathrm{~Hz}, 3 \mathrm{H}), 0.87(\mathrm{t}, J=6.9 \mathrm{~Hz}, 3 \mathrm{H}) ;{ }^{13} \mathrm{C} \mathrm{NMR}\left(101 \mathrm{MHz}, \mathrm{CDCl}_{3}\right) \delta 139.3,128.6,83.2,75.7$, 69.4, 39.1, 38.2, 36.3, 22.1, 20.5, 20.4, 14.5, 14.1; IR (neat) 3420 (br), 3312, 2949, 2361, 1456, $989 \mathrm{~cm}^{-1}$; EIMS m/z 163 (29), 149 (36), 105 (100), 77 (38), 69 (34); HREIMS calcd for $\mathrm{C}_{8} \mathrm{H}_{15} \mathrm{O}$ $\left(\mathrm{M}^{+}-\mathrm{HC} \equiv \mathrm{CCH}_{2}\left(\mathrm{CH}_{3}\right) \mathrm{CH} \cdot\right)$ 127.1123, found 127.1119; optical rotation $[\alpha]^{27}{ }_{\mathrm{D}}+41^{\circ}(c$ 1.0, $\mathrm{CH}_{2} \mathrm{Cl}_{2}$ ). 


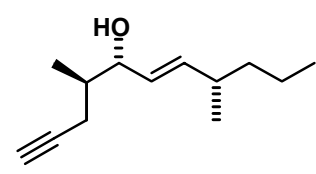

31

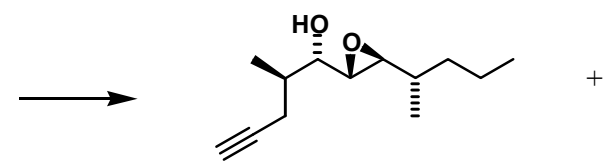

20

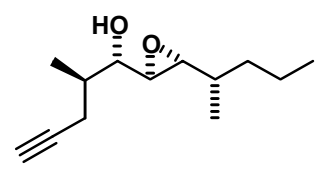

32

To a solution of alkene $31(272 \mathrm{mg}, 1.39 \mathrm{mmol})$ in $\mathrm{CH}_{2} \mathrm{Cl}_{2}(11 \mathrm{~mL})$ at $-10^{\circ} \mathrm{C}$ was added $\mathrm{VO}(\mathrm{acac})_{2}(26 \mathrm{mg}, 0.098 \mathrm{mmol})$ and tert-butyl hydroperoxide $(840 \mu \mathrm{L}, 5-6 \mathrm{M}$ in decane, 4.2-5.0 mmol). The reaction mixture was stirred at $-10^{\circ} \mathrm{C}$ for $6 \mathrm{~h}$ and quenched with saturated $\mathrm{Na}_{2} \mathrm{SO}_{3}$. After stirring for $15 \mathrm{~min}$, the reaction mixture was diluted with ether and water. The aqueous phase was extracted with ether $(3 \mathrm{x})$ and the combined organic extracts were washed with brine $(1 \mathrm{x})$, dried over $\mathrm{MgSO}_{4}$, and concentrated. Purification by flash column chromatography on silica gel (5\% to $7.5 \%$ to $10 \%$ to $15 \%$ to $20 \%$ EtOAc in petroleum ether) gave a mixture of epoxides 20 (231 mg, 78\%) and 32 (45 mg, 15\%) as colorless oils. Data for 20: $\mathrm{R}_{f}=0.44(20 \%$ EtOAc in petroleum ether); ${ }^{1} \mathrm{H} \mathrm{NMR}\left(400 \mathrm{MHz}, \mathrm{CDCl}_{3}\right) \delta 3.92(\mathrm{t}, J=3.5 \mathrm{~Hz}, 1 \mathrm{H}), 2.85(\mathrm{dd}, J=$ 6.9, $1.8 \mathrm{~Hz}, 1 \mathrm{H}), 2.82$ (t, $J=2.9 \mathrm{~Hz}, 1 \mathrm{H}), 2.39$ (ddd, $J=16.8,6.9,2.6 \mathrm{~Hz}, 1 \mathrm{H}), 2.23$ (ddd, $J=$ 16.8, 7.2, $2.8 \mathrm{~Hz}, 1 \mathrm{H}), 2.00(\mathrm{dd}, J=2.6,2.1 \mathrm{~Hz}, 1 \mathrm{H}), 1.94(\mathrm{~m}, 1 \mathrm{H}), 1.54-1.19(\mathrm{~m}, 6 \mathrm{H}), 1.07(\mathrm{~d}, J$ $=6.9 \mathrm{~Hz}, 3 \mathrm{H}), 0.95(\mathrm{~d}, J=6.9 \mathrm{~Hz}, 3 \mathrm{H}), 0.91(\mathrm{t}, J=7.2 \mathrm{~Hz}, 3 \mathrm{H}) ;{ }^{13} \mathrm{C} \mathrm{NMR}\left(101 \mathrm{MHz}, \mathrm{CDCl}_{3}\right) \delta$ 82.8, 70.3, 67.9, 59.8, 58.2, 36.7, 35.7, 35.2, 22.8, 20.0, 15.9, 14.2, 14.0; IR (neat) 3466 (br), 3312, 2959, 2361, $1460 \mathrm{~cm}^{-1}$; CIMS $m / z 211.2[\mathrm{M}+\mathrm{H}]^{+}$; optical rotation $[\alpha]^{26}{ }_{\mathrm{D}}+2.0^{\circ}(c 0.25$, $\mathrm{CH}_{2} \mathrm{Cl}_{2}$ ); Anal. Calcd for $\mathrm{C}_{13} \mathrm{H}_{22} \mathrm{O}_{2}$ : C, 74.24; $\mathrm{H}, 10.54$. Found: $\mathrm{C}, 74.24 ; \mathrm{H}, 10.68$. Data for 32: $\mathrm{R}_{f}=0.39\left(20 \%\right.$ EtOAc in petroleum ether); ${ }^{1} \mathrm{H} \mathrm{NMR}\left(400 \mathrm{MHz}, \mathrm{CDCl}_{3}\right) \delta 3.64(\mathrm{t}, J=4.4 \mathrm{~Hz}$, 1H), 2.91 (dd, $J=4.1,2.4 \mathrm{~Hz}, 1 \mathrm{H}), 2.75$ (dd, $J=6.9,2.3 \mathrm{~Hz}, 1 \mathrm{H}), 2.37$ (ddd, $J=16.8,6.9,2.6$ $\mathrm{Hz}, 1 \mathrm{H}), 2.24$ (ddd, $J=16.8,6.6,2.4 \mathrm{~Hz}, 1 \mathrm{H}), 2.00$ (t, $J=2.4 \mathrm{~Hz}, 1 \mathrm{H}), 1.93$ (m, 1H), 1.43-1.20 $(\mathrm{m}, 6 \mathrm{H}), 1.09(\mathrm{~d}, J=6.9 \mathrm{~Hz}, 3 \mathrm{H}), 1.01(\mathrm{~d}, J=6.4 \mathrm{~Hz}, 3 \mathrm{H}), 0.90(\mathrm{t}, J=6.9 \mathrm{~Hz}, 3 \mathrm{H}) ;{ }^{13} \mathrm{C} \mathrm{NMR}$ $\left(101 \mathrm{MHz}, \mathrm{CDCl}_{3}\right) \delta 82.7,72.1,69.7,61.0,60.1,37.1,35.5,35.1,22.6,20.2,16.8,14.2,14.1$; IR (neat) 3464 (br), 3310, 2951, 2353, 1462, $902 \mathrm{~cm}^{-1}$; CIMS m/z $211.2[\mathrm{M}+\mathrm{H}]^{+}$; optical rotation $[\alpha]^{27}{ }_{D}+39^{\circ}\left(c 0.50, \mathrm{CH}_{2} \mathrm{Cl}_{2}\right)$; Anal. Calcd for $\mathrm{C}_{13} \mathrm{H}_{22} \mathrm{O}_{2}$ : C, 74.24; H, 10.54. Found: C, 73.99; H, 10.44 .

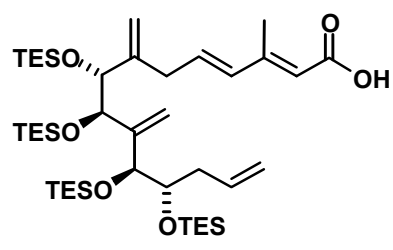

19

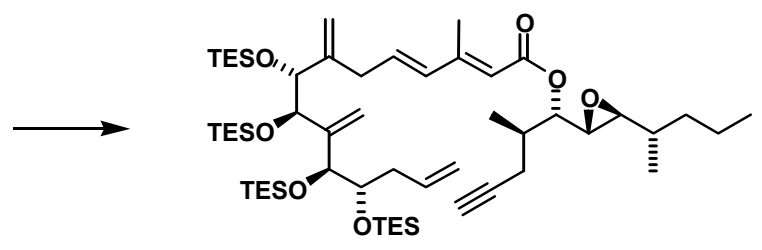

21 
To a solution of acid $19(170 \mathrm{mg}, 0.21 \mathrm{mmol})$ in $\mathrm{PhMe}(3.0 \mathrm{~mL})$ at room temperature was added $\left[\mathrm{RuCl}_{2}(p \text {-cymene })\right]_{2}(12 \mathrm{mg}, 0.020 \mathrm{mmol})$ and ethoxyacetylene $(60 \mu \mathrm{L}, 50 \% \mathrm{wt}$. in hexanes, $0.34 \mathrm{mmol}$ ). The reaction mixture was stirred at room temperature for $2 \mathrm{~h}$ and concentrated under a stream of argon. A solution of alcohol 20 (108 mg, $0.51 \mathrm{mmol})$ in DCE (2.5 $\mathrm{mL})$ was added via cannula followed by CSA $(5.1 \mathrm{mg}, 22 \mu \mathrm{mol})$. The reaction mixture was stirred at room temperature for $2 \mathrm{~h}$, filtered through silica gel, and concentrated. Purification by flash column chromatography on silica gel (1\% to $2 \%$ to $5 \%$ to $20 \%$ EtOAc in petroleum ether) gave ester 21 (108 mg, 51\%) as a colorless oil: $\mathrm{R}_{f}=0.36\left(5 \%\right.$ EtOAc in petroleum ether); ${ }^{1} \mathrm{H}$ $\operatorname{NMR}\left(500 \mathrm{MHz}, \mathrm{CDCl}_{3}\right) \delta$ 6.20-6.10 (m, 2H), $5.82(\mathrm{dddd}, J=17.2,14.4,10.3,7.3 \mathrm{~Hz}, 1 \mathrm{H}), 5.71$ (s, 1H), 5.40-5.39 (m, 2H), $5.08(\mathrm{~s}, 1 \mathrm{H}), 5.05(\mathrm{dm}, J=9.0 \mathrm{~Hz}, 1 \mathrm{H}), 5.02(\mathrm{~d}, J=1.2 \mathrm{~Hz}, 1 \mathrm{H}), 4.79$ $(\mathrm{d}, J=1.5 \mathrm{~Hz}, 1 \mathrm{H}), 4.70(\mathrm{dd}, J=6.7,4.3 \mathrm{~Hz}, 1 \mathrm{H}), 4.26(\mathrm{~d}, J=4.0 \mathrm{~Hz}, 1 \mathrm{H}), 4.23(\mathrm{~d}, J=3.7 \mathrm{~Hz}$, $1 \mathrm{H}), 4.21(\mathrm{~d}, J=3.5 \mathrm{~Hz}, 1 \mathrm{H}), 3.80(\mathrm{ddd}, J=8.2,3.9,3.9 \mathrm{~Hz}, 1 \mathrm{H}), 3.00(\mathrm{dd}, J=16.6,5.7 \mathrm{~Hz}$, 1H), $2.94(\mathrm{dd}, J=17.2,6.4 \mathrm{~Hz}, 1 \mathrm{H}), 2.82(\mathrm{dd}, J=7.5,2.2 \mathrm{~Hz}, 1 \mathrm{H}), 2.75(\mathrm{dd}, J=6.7,2.1 \mathrm{~Hz}$, 1H), $2.40(\mathrm{dm}, J=14.2 \mathrm{~Hz}, 1 \mathrm{H}), 2.34(\mathrm{ddd}, J=15.9,5.5,2.7 \mathrm{~Hz}, 1 \mathrm{H}), 2.29(\mathrm{~d}, J=1.1 \mathrm{~Hz}, 3 \mathrm{H})$, $2.19(\mathrm{dd}, J=7.9,2.7 \mathrm{~Hz}, 1 \mathrm{H}), 2.16-2.11(\mathrm{~m}, 2 \mathrm{H}), 1.98(\mathrm{t}, J=2.7 \mathrm{~Hz}, 1 \mathrm{H}), 1.49-1.22(\mathrm{~m}, 5 \mathrm{H})$, $1.14(\mathrm{~d}, J=6.6 \mathrm{~Hz}, 3 \mathrm{H}), 0.98-0.92(\mathrm{~m}, 39 \mathrm{H}), 0.88(\mathrm{t}, J=6.7 \mathrm{~Hz}, 3 \mathrm{H}), 0.65-0.55(\mathrm{~m}, 24 \mathrm{H}) ;{ }^{13} \mathrm{C}$ NMR $\left(126 \mathrm{MHz}, \mathrm{CDCl}_{3}\right) \delta 166.1,153.5,148.8,146.5,136.5,135.5,135.0,117.2,117.0,116.6$, $112.8,82.3,78.0,75.9,74.9,74.2,69.9,69.6,62.6,55.7,37.1,36.6,35.4,35.2,35.0,22.4,20.0$, $15.7,15.6,14.3,14.2,7.12,7.09,7.06,7.0,5.4,5.30,5.27,5.1$; IR (neat) 3312, 2946, 1714, 1605, 1146, 1094, $741 \mathrm{~cm}^{-1}$; ESIMS $m / z 987.1[\mathrm{M}+\mathrm{H}]^{+}$; optical rotation $[\alpha]^{23} \mathrm{D}-18^{\circ}(c 0.50$, $\mathrm{CH}_{2} \mathrm{Cl}_{2}$ ).
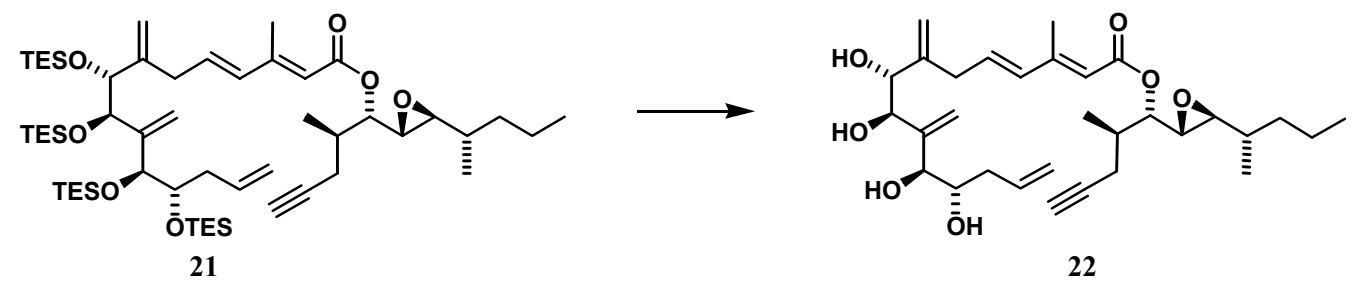

To a solution of silyl ether $21(45 \mathrm{mg}, 0.046 \mathrm{mmol})$ in THF $(1.0 \mathrm{~mL})$ at room temperature was added $200 \mu \mathrm{L}$ of a mixture of TBAF $(2.0 \mathrm{~mL}, 1 \mathrm{M}$ in THF, $2.0 \mathrm{mmol})$ and acetic acid (115 $\mu \mathrm{L}, 121 \mathrm{mg}, 2.01 \mathrm{mmol})$. The reaction mixture was stirred at room temperature for $30 \mathrm{~h}$ and concentrated. Purification by flash column chromatography on silica gel (30\% to $50 \%$ to $70 \%$ EtOAc in petroleum ether) gave tetraol $22(19 \mathrm{mg}, 79 \%)$ as an amorphous solid: $\mathrm{R}_{f}=0.54$ 
(EtOAc); ${ }^{1} \mathrm{H}$ NMR $\left(500 \mathrm{MHz}, \mathrm{CDCl}_{3}\right) \delta$ 6.19-6.14 (m, 2H), 5.85 (dddd, $J=16.8,14.3,10.6,7.1$ $\mathrm{Hz}, 1 \mathrm{H}), 5.72(\mathrm{~d}, J=1.0 \mathrm{~Hz}, 1 \mathrm{H}), 5.34$ (s, 1H), 5.32 (s, 1H), 5.23 (s, 1H), 5.17 (dm, $J=7.2 \mathrm{~Hz}$, $1 \mathrm{H}), 5.14(\mathrm{dd}, J=1.1,1.1 \mathrm{~Hz}, 1 \mathrm{H}), 5.02(\mathrm{~d}, J=1.0 \mathrm{~Hz}, 1 \mathrm{H}), 4.71(\mathrm{dd}, J=4.2,2.1 \mathrm{~Hz}, 1 \mathrm{H}), 4.33-$ $4.31(\mathrm{~m}, 2 \mathrm{H}), 4.17$ (d, $J=4.9 \mathrm{~Hz}, 1 \mathrm{H}), 3.83$ (ddd, $J=8.3,4.6,4.6 \mathrm{~Hz}, 1 \mathrm{H}), 3.03$ (dd, $J=17.0$, $4.9 \mathrm{~Hz}, 1 \mathrm{H}), 2.92(\mathrm{dd}, J=16.6,4.4 \mathrm{~Hz}, 1 \mathrm{H}), 2.80(\mathrm{dd}, J=7.5,2.1 \mathrm{~Hz}, 1 \mathrm{H}), 2.75(\mathrm{dd}, J=6.5,2.2$ Hz, 1H), 2.39 (dm, $J=14.2 \mathrm{~Hz}, 1 \mathrm{H}$ ), 2.33 (ddd, $J=16.2,5.6,2.8 \mathrm{~Hz}, 1 \mathrm{H}$ ), 2.26 (d, $J=1.1 \mathrm{~Hz}$, $3 \mathrm{H}$ ), 2.25 (dd, $J=14.5,8.1 \mathrm{~Hz}, 1 \mathrm{H}), 2.18(\mathrm{dd}, J=4.3,2.7 \mathrm{~Hz}, 1 \mathrm{H}), 2.13(\mathrm{~m}, 1 \mathrm{H}), 1.98$ (t, $J=2.7$ $\mathrm{Hz}, 1 \mathrm{H}), 1.48-1.21(\mathrm{~m}, 5 \mathrm{H}), 1.13$ (d, $J=6.7 \mathrm{~Hz}, 3 \mathrm{H}), 0.88$ (t, $J=7.2 \mathrm{~Hz}, 3 \mathrm{H}), 0.87$ (d, $J=6.7$ $\mathrm{Hz}, 3 \mathrm{H}) ;{ }^{13} \mathrm{C} \mathrm{NMR}\left(126 \mathrm{MHz}, \mathrm{CDCl}_{3}\right) \delta 166.0,152.9,147.5,146.3,135.6,134.24,134.18$, 118.5, 117.8, 116.7, 114.0, 82.2, 76.0, 74.8, 74.1, 73.0, 72.3, 69.8, 62.5, 55.6, 37.9, 36.6, 36.0, 35.2, 35.0, 22.5, 19.9, 15.6, 14.3, 14.2, 14.0; IR (neat) 3424 (br), 3297, 2917, 1716, 1607, 1236, $1150 \mathrm{~cm}^{-1}$; ESIMS $m / z 553.3[\mathrm{M}+\mathrm{Na}]^{+}$; optical rotation $[\alpha]^{29}{ }_{\mathrm{D}}+4.8^{\circ}\left(c 0.10, \mathrm{CH}_{2} \mathrm{Cl}_{2}\right)$.
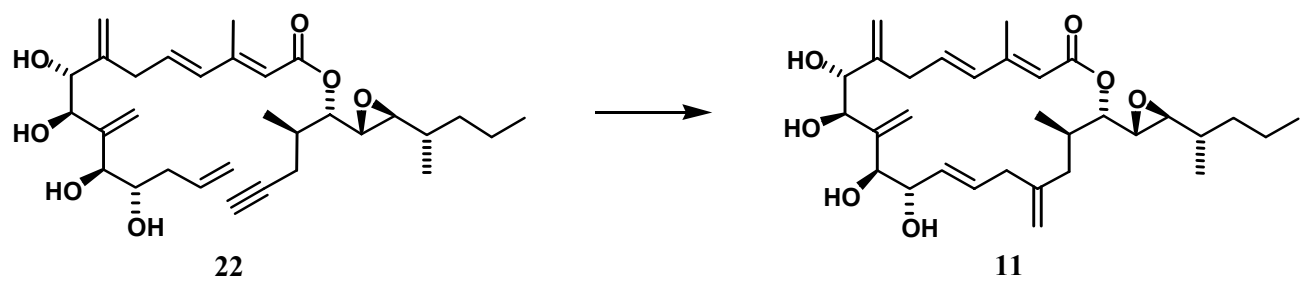

A solution of alkyne $22(15.6 \mathrm{mg}, 0.029 \mathrm{mmol})$ in DCE $(30 \mathrm{~mL})$ was degassed by bubbling nitrogen through the solution for $15 \mathrm{~min}$. The reaction mixture was heated to $50^{\circ} \mathrm{C}$ and $\mathrm{Cp} * \mathrm{Ru}(\mathrm{MeCN})_{3} \mathrm{PF}_{6}(3.6 \mathrm{mg}, 7.1 \mu \mathrm{mol})$ was added. After $6 \mathrm{~h}$ at $50^{\circ} \mathrm{C}$, the reaction mixture was filtered through silica gel, and concentrated. Purification by flash column chromatography on silica gel (40\% to $60 \%$ to $80 \%$ EtOAc in petroleum ether) gave diene $11(5.2 \mathrm{mg}, 33 \%)$ and recovered alkyne $22(1.9 \mathrm{mg}, 12 \%)$ as amorphous solids. Data for 11: $\mathrm{R}_{f}=0.33(80 \%$ EtOAc in petroleum ether); ${ }^{1} \mathrm{H}$ NMR (500 MHz, $\left.\mathrm{CDCl}_{3}\right) \delta 6.28(\mathrm{~d}, J=15.4 \mathrm{~Hz}, 1 \mathrm{H}), 6.10$ (ddd, $J=15.5$, 9.6, $5.1 \mathrm{~Hz}, 1 \mathrm{H}$ ), 5.80 (s, 1H), 5.69 (dddd, $J=15.3,7.3,7.3,1.5 \mathrm{~Hz}, 1 \mathrm{H}$ ), 5.50 (dd, $J=15.4,4.8$ $\mathrm{Hz}, 1 \mathrm{H}), 5.49(\mathrm{~s}, 1 \mathrm{H}), 5.38(\mathrm{~d}, J=1.0 \mathrm{~Hz}, 1 \mathrm{H}), 5.36(\mathrm{~d}, J=1.0 \mathrm{~Hz}, 1 \mathrm{H}), 5.21(\mathrm{~s}, 1 \mathrm{H}), 4.88(\mathrm{~s}$, 1H), 4.79 (s, 1H), 4.72 (dd, $J=6.1,3.2 \mathrm{~Hz}, 1 \mathrm{H}), 4.59$ (s br, 1H), 4.43 (s br, 1H), 4.22 (s br, 1H), 4.06 ( $\mathrm{br}, J=3.8 \mathrm{~Hz}, 1 \mathrm{H}), 3.21$ (dd, $J=14.9,5.0 \mathrm{~Hz}, 1 \mathrm{H}), 3.11$ (dd, $J=14.7,9.3 \mathrm{~Hz}, 1 \mathrm{H}), 2.85$ (dd, $J=6.2,2.2 \mathrm{~Hz}, 1 \mathrm{H}), 2.76$ (dd, $J=7.5,2.2 \mathrm{~Hz}, 1 \mathrm{H}), 2.76$ (s br, 2H), 2.34 (dd, $J=15.3,5.0$ $\mathrm{Hz}, 1 \mathrm{H}), 2.27$ (d, $J=1.1 \mathrm{~Hz}, 3 \mathrm{H}), 2.16(\mathrm{~m}, 1 \mathrm{H}), 1.92$ (dd, $J=14.4,9.2 \mathrm{~Hz}, 1 \mathrm{H}), 1.53-1.24$ (m, $5 \mathrm{H}), 1.06(\mathrm{~d}, J=7.0 \mathrm{~Hz}, 3 \mathrm{H}), 0.93(\mathrm{~d}, J=6.8 \mathrm{~Hz}, 3 \mathrm{H}), 0.91(\mathrm{t}, J=7.1 \mathrm{~Hz}, 3 \mathrm{H}) ;{ }^{13} \mathrm{C} \mathrm{NMR}(101$ 
$\left.\mathrm{MHz}, \mathrm{CDCl}_{3}\right) \delta 165.8,152.8,147.2,145.0,144.8,136.4,134.8,130.9,130.6,118.6,116.1$, 114.7, 112.9, 75.8, 74.7, 73.5, 72.5, 70.5, 61.8, 54.2, 39.7, 39.0, 36.7, 36.2, 35.4, 33.3, 20.0, 15.8, 14.9, 14.3, 13.9; IR (neat) 3438 (br), 2927, 1715, 1612, 1235, 1150, $850 \mathrm{~cm}^{-1}$; ESIMS $\mathrm{m} / z 553.3$ $[\mathrm{M}+\mathrm{Na}]^{+}$; HRESIMS calcd for $\mathrm{C}_{31} \mathrm{H}_{46} \mathrm{O}_{7} \mathrm{Na}\left[\mathrm{M}^{+}+\mathrm{Na}\right]$ 553.3141, found 553.3157; optical rotation $[\alpha]^{24}+56^{\circ}\left(c 0.05, \mathrm{CHCl}_{3}\right)$.

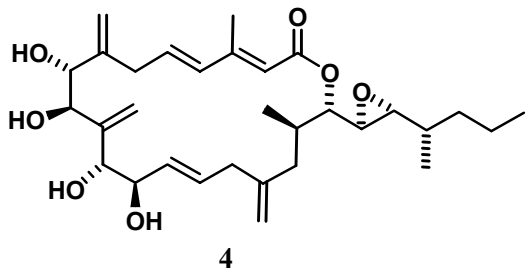

Data for 4: $\mathrm{R}_{f}=0.31(\mathrm{EtOAc}) ;{ }^{1} \mathrm{H}$ NMR $\left(500 \mathrm{MHz}, \mathrm{CDCl}_{3}\right) \delta 6.19(\mathrm{~d}, J=15.6 \mathrm{~Hz}, 1 \mathrm{H})$, 6.08 (ddd, $J=14.9,9.8,3.8 \mathrm{~Hz}, 1 \mathrm{H}), 5.79(\mathrm{~s}, 1 \mathrm{H}), 5.77-5.72(\mathrm{~m}, 1 \mathrm{H}), 5.59(\mathrm{~s}, 1 \mathrm{H}), 5.57$ (s, 1H), 5.37 (s, 1H), $5.26(\mathrm{~d} \mathrm{br}, J=16.2 \mathrm{~Hz}, 1 \mathrm{H}), 5.22(\mathrm{~s}, 1 \mathrm{H}), 4.86(\mathrm{~s}, 1 \mathrm{H}), 4.78$ (s, 1H), 4.61 (dd, $J=$ 6.2, $3.8 \mathrm{~Hz}, 1 \mathrm{H}), 4.28(\mathrm{~s}, 1 \mathrm{H}), 4.23$ (s, 1H), 4.20 (s, 1H), 3.83 (s, 1H), 3.22 (dd, $J=15.1,4.4 \mathrm{~Hz}$, 1H), 3.11 (dd, $J=15.3,10.4 \mathrm{~Hz}, 1 \mathrm{H}), 2.97$ (dd, $J=6.1,2.1 \mathrm{~Hz}, 1 \mathrm{H}), 2.76$ (dd, $J=13.6,4.0 \mathrm{~Hz}$, $1 \mathrm{H}), 2.69(\mathrm{dd}, J=8.9,2.1 \mathrm{~Hz}, 1 \mathrm{H}), 2.66(\mathrm{dd}, J=14.2,10.4 \mathrm{~Hz}, 1 \mathrm{H}), 2.51(\mathrm{dd}, J=12.5,2.5 \mathrm{~Hz}$, 1H), 2.29 (s, 3H), $2.13(\mathrm{~m}, 1 \mathrm{H}), 1.79$ (dd, $J=12.7,11.6 \mathrm{~Hz}, 1 \mathrm{H}), 1.44-1.22(\mathrm{~m}, 5 \mathrm{H}), 1.01$ (d, $J=$ $6.4 \mathrm{~Hz}, 3 \mathrm{H}), 0.99(\mathrm{~d}, J=7.0 \mathrm{~Hz}, 3 \mathrm{H}), 0.91(\mathrm{t}, J=7.0 \mathrm{~Hz}, 3 \mathrm{H})$, exchangeable protons not listed; ${ }^{13} \mathrm{C}$ NMR $\left(126 \mathrm{MHz}, \mathrm{CDCl}_{3}\right) \delta 165.6,152.1,146.6,145.2,143.7,136.3,133.8,131.2,130.2$, 119.3, 115.4, 114.8, 110.6, 78.8, 76.4, 72.5, 69.6, 65.9, 61.0, 52.6, 41.3, 38.6, 36.4, 35.3, 35.2, 33.4, 20.2, 15.9, 15.3, 14.2, 13.6; IR (neat) 3293 (br), 1709, 1234, $1152 \mathrm{~cm}^{-1}$; ESIMS m/z 553.3 $[\mathrm{M}+\mathrm{Na}]^{+}$; optical rotation $[\alpha]^{23}{ }_{\mathrm{D}}+55^{\circ}\left(c 0.10, \mathrm{CH}_{2} \mathrm{Cl}_{2}\right)$.

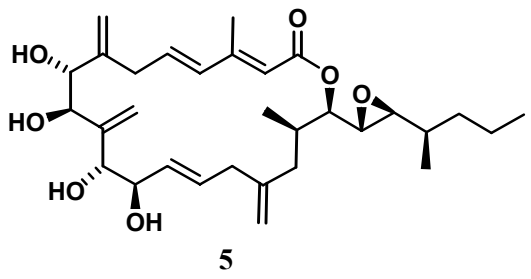

Data for 5: $\mathrm{R}_{f}=0.38(\mathrm{EtOAc}){ }^{1} \mathrm{H}$ NMR $\left(500 \mathrm{MHz}, \mathrm{CDCl}_{3}\right) \delta 6.19(\mathrm{~d}, J=15.4 \mathrm{~Hz}, 1 \mathrm{H})$, 6.08 (ddd, $J=14.7,8.1,6.4 \mathrm{~Hz}, 1 \mathrm{H}), 5.81$ (s, $1 \mathrm{H}), 5.72$ (dddd, $J=15.4,8.6,7.0,1.2 \mathrm{~Hz}, 1 \mathrm{H}$ ), 5.49 (s, 1H), $5.48(\mathrm{ddm}, J=15.6,4.9 \mathrm{~Hz}, 1 \mathrm{H}), 5.44(\mathrm{~s}, 1 \mathrm{H}), 5.34(\mathrm{~s}, 1 \mathrm{H}), 5.24(\mathrm{~s}, 1 \mathrm{H}), 4.83(\mathrm{~s}$, 1H), $4.75(\mathrm{~s}, 1 \mathrm{H}), 4.60(\mathrm{dd}, J=10.3,5.5 \mathrm{~Hz}, 1 \mathrm{H}), 4.32(\mathrm{~s}, 1 \mathrm{H}), 4.16(\mathrm{~s}, 1 \mathrm{H}), 4.09(\mathrm{~m}, 1 \mathrm{H}), 3.95$ 
(s, 1H), 3.25 (dd, $J=14.4,6.4 \mathrm{~Hz}, 1 \mathrm{H}), 3.02(\mathrm{dd}, J=5.6,2.2 \mathrm{~Hz}, 1 \mathrm{H}), 3.00(\mathrm{dd}, J=14.4,8.2 \mathrm{~Hz}$, 1H), 2.76-2.74 (m, 2H), 2.72 (dd, $J=6.7,2.1 \mathrm{~Hz}, 1 \mathrm{H}), 2.26$ (d, $J=1.0 \mathrm{~Hz}, 3 \mathrm{H}), 2.17$ (dd, $J=$ 12.9, $2.6 \mathrm{~Hz}, 1 \mathrm{H}), 1.94(\mathrm{~m}, 1 \mathrm{H}), 1.65(\mathrm{dd}, J=13.3,9.6 \mathrm{~Hz}, 1 \mathrm{H}), 1.47-1.21(\mathrm{~m}, 5 \mathrm{H}), 1.04(\mathrm{~d}, J=$ $6.7 \mathrm{~Hz}, 3 \mathrm{H}), 1.00(\mathrm{~d}, J=6.6 \mathrm{~Hz}, 3 \mathrm{H}), 0.92(\mathrm{t}, J=7.2 \mathrm{~Hz}, 3 \mathrm{H})$, exchangeable protons not listed; ${ }^{13} \mathrm{C}$ NMR $\left(101 \mathrm{MHz}, \mathrm{CDCl}_{3}\right) \delta 165.9,152.1,146.9,145.0,144.9,135.6,134.7,131.7,130.2$, 118.5, 115.6, 115.0, 113.5, 77.2, 76.1, 74.8, 74.3, 72.5, 72.3, 62.4, 58.4, 39.5, 39.2, 38.8, 35.4, 35.0, 33.6, 20.0, 16.4, 15.7, 14.2; IR (neat) 3286 (br), 2963, 1706, 1616, $1150 \mathrm{~cm}^{-1}$; ESIMS $\mathrm{m} / \mathrm{z}$ $553.3[\mathrm{M}+\mathrm{Na}]^{+}$; optical rotation $[\alpha]^{23}-65^{\circ}\left(c 0.10, \mathrm{CH}_{2} \mathrm{Cl}_{2}\right)$.

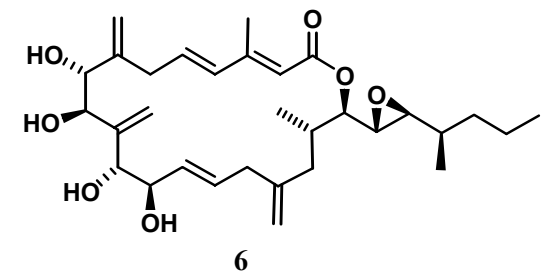

Data for 6: $\mathrm{R}_{f}=0.30$ (EtOAc); ${ }^{1} \mathrm{H}$ NMR $\left(500 \mathrm{MHz}, \mathrm{CDCl}_{3}\right) \delta 6.16(\mathrm{~d}, J=15.9 \mathrm{~Hz}, 1 \mathrm{H})$, 6.08 (ddd, $J=15.5,7.0,6.7 \mathrm{~Hz}, 1 \mathrm{H}), 5.82(\mathrm{~s}, 1 \mathrm{H}), 5.68$ (ddd, $J=15.4,7.8,6.6,1.2 \mathrm{~Hz}, 1 \mathrm{H}), 5.47$ (s, 1H), 5.39 (s, 1H), 5.38 (dd, $J=15.4,5.4 \mathrm{~Hz}, 1 \mathrm{H}), 5.32$ (s, 1H), 5.20 (s, 1H), 4.85 (s br, 1H), 4.77 (s br, 1H), 4.67 (dd, $J=6.1,3.4 \mathrm{~Hz}, 1 \mathrm{H}), 4.38$ (d, $J=4.3 \mathrm{~Hz}, 1 \mathrm{H}), 4.17$ (dd, $J=4.0,3.7 \mathrm{~Hz}$, $1 \mathrm{H}), 4.10$ (d, $J=4.3 \mathrm{~Hz}, 1 \mathrm{H}), 3.89$ (d, $J=3.5 \mathrm{~Hz}, 1 \mathrm{H}), 3.21$ (dd, $J=14.7,6.5 \mathrm{~Hz}, 1 \mathrm{H}), 2.98$ (dd, $J=14.5,7.4 \mathrm{~Hz}, 1 \mathrm{H}), 2.97(\mathrm{dd}, J=6.1,2.2 \mathrm{~Hz}, 1 \mathrm{H}), 2.73(\mathrm{dd}, J=13.9,6.0 \mathrm{~Hz}, 1 \mathrm{H}), 2.66(\mathrm{dd}, J$ $=7.0,2.2 \mathrm{~Hz}, 1 \mathrm{H}), 2.63(\mathrm{dd}, J=14.7,8.3 \mathrm{~Hz}, 1 \mathrm{H}), 2.40(\mathrm{dd}, J=13.7,3.9 \mathrm{~Hz}, 1 \mathrm{H}), 2.25(\mathrm{~d}, J=$ $1.1 \mathrm{~Hz}, 3 \mathrm{H}), 2.11$ (m, 1H), 1.82 (dd, $J=13.7,10.1 \mathrm{~Hz}, 1 \mathrm{H}), 1.43-1.20$ (m, 5H), 1.01 (d, $J=7.0$ $\mathrm{Hz}, 3 \mathrm{H}), 1.00(\mathrm{~d}, J=6.5 \mathrm{~Hz}, 3 \mathrm{H}), 0.91(\mathrm{t}, J=7.0 \mathrm{~Hz}, 3 \mathrm{H})$, exchangeable protons not listed; ${ }^{13} \mathrm{C}$ $\operatorname{NMR}\left(125 \mathrm{MHz}, \mathrm{CDCl}_{3}\right) \delta 166.0,152.4,147.0,145.1,144.7,133.4,134.9,131.5,130.3,118.3$, 116.2, 115.0, 113.4, 76.4, 75.1, 74.1, 73.6, 72.2, 60.9, 55.8, 39.2, 38.3, 36.4, 35.4, 35.2, 34.1, 20.2, 16.7, 15.7, 14.4, 14.2; IR (neat) 3285, 2922, 1709, 1614, $1153 \mathrm{~cm}^{-1}$; ESIMS $\mathrm{m} / z 553.4$ [M $+\mathrm{Na}]^{+}$; optical rotation $[\alpha]^{26}{ }_{\mathrm{D}}-42^{\circ}\left(c 0.10, \mathrm{CH}_{2} \mathrm{Cl}_{2}\right)$.

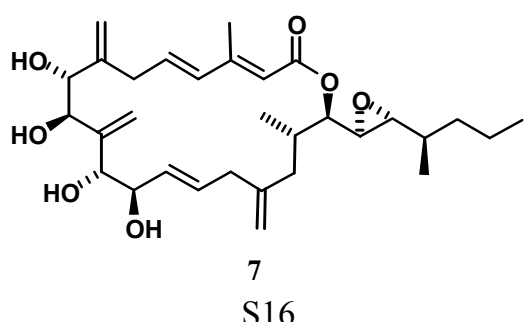


Data for 7: $\mathrm{R}_{f}=0.41\left(10 \% \mathrm{MeOH}\right.$ in $\left.\mathrm{CH}_{2} \mathrm{Cl}_{2}\right) ;{ }^{1} \mathrm{H}$ NMR $\left(500 \mathrm{MHz}, \mathrm{CDCl}_{3}\right) \delta 6.16(\mathrm{~d}, J=$ $15.5 \mathrm{~Hz}, 1 \mathrm{H}), 6.08$ (ddd, $J=15.9,6.8,6.5 \mathrm{~Hz}, 1 \mathrm{H}), 5.77$ (s, 1H), 5.69 (ddd, $J=14.2,6.3,6.3 \mathrm{~Hz}$, 1H), $5.48(\mathrm{~s}, 1 \mathrm{H}), 5.41(\mathrm{dd}, J=15.5,5.4 \mathrm{~Hz}, 1 \mathrm{H}), 5.39(\mathrm{~s}, 1 \mathrm{H}), 5.33(\mathrm{~s}, 1 \mathrm{H}), 5.21(\mathrm{~s}, 1 \mathrm{H}), 4.87$ (s, 1H), 4.79 (s, 1H), 4.67 (dd, $J=6.4,3.2 \mathrm{~Hz}, 1 \mathrm{H}), 4.38$ (s br, 1H), 4.18 (s br, 1H), 4.12 (s br, 1H), 3.91 (s br, 1H), 3.21 (dd, $J=14.9,7.0 \mathrm{~Hz}, 1 \mathrm{H}), 2.98$ (dd, $J=14.4,7.1 \mathrm{~Hz}, 1 \mathrm{H}), 2.84$ (dd, $J=6.2$, $2.1 \mathrm{~Hz}, 1 \mathrm{H}), 2.77(\mathrm{dd}, J=7.6,2.2 \mathrm{~Hz}, 1 \mathrm{H}), 2.74(\mathrm{dd}, J=14.8,6.7 \mathrm{~Hz}, 1 \mathrm{H}), 2.66$ (dd, $J=14.8$, $7.3 \mathrm{~Hz}, 1 \mathrm{H}), 2.26(\mathrm{~d} \mathrm{br}, J=14.0 \mathrm{~Hz}, 1 \mathrm{H}), 2.24(\mathrm{~d}, J=1.0 \mathrm{~Hz}, 3 \mathrm{H}), 2.14(\mathrm{~m}, 1 \mathrm{H}), 1.88(\mathrm{dd}, J=$ 13.9, $9.2 \mathrm{~Hz}, 1 \mathrm{H}), 1.51-1.25(\mathrm{~m}, 5 \mathrm{H}), 1.05(\mathrm{~d}, J=7.1 \mathrm{~Hz}, 3 \mathrm{H}), 0.91$ (t, $J=7.0 \mathrm{~Hz}, 3 \mathrm{H}), 0.89$ (d, $J$ $=7.1 \mathrm{~Hz}, 3 \mathrm{H})$.

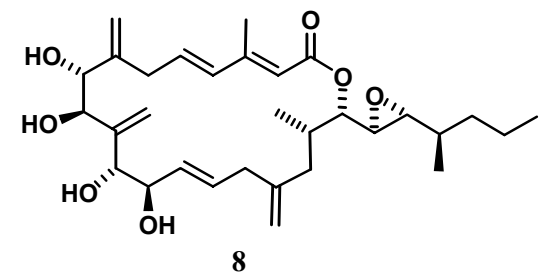

Data for 8: $\mathrm{R}_{f}=0.38(\mathrm{EtOAc}) ;{ }^{1} \mathrm{H} \mathrm{NMR}\left(500 \mathrm{MHz}, \mathrm{CDCl}_{3}\right) \delta 6.16(\mathrm{~d}, J=15.6 \mathrm{~Hz}, 1 \mathrm{H})$, 6.03 (ddd, $J=14.9,9.8,4.8 \mathrm{~Hz}, 1 \mathrm{H}), 5.75$ (ddd, $J=15.3,7.3,6.4 \mathrm{~Hz}, 1 \mathrm{H}), 5.72$ (s, 1H), 5.53 (dd, $J=15.6,5.4 \mathrm{~Hz}, 1 \mathrm{H}), 5.49(\mathrm{~s}, 1 \mathrm{H}), 5.42(\mathrm{~s}, 1 \mathrm{H}), 5.33(\mathrm{~s}, 1 \mathrm{H}), 5.20(\mathrm{~s}, 1 \mathrm{H}), 4.74(\mathrm{~s}, 1 \mathrm{H}), 4.73$ (s, 1H), 4.56 (dd, $J=10.3,5.6 \mathrm{~Hz}, 1 \mathrm{H}), 4.36$ (s br, 1H), 4.30 (s br, 1H), 4.27 (s br, 1H), 3.99 (s br, $1 \mathrm{H}), 3.16(\mathrm{dd}, J=14.4,4.3 \mathrm{~Hz}, 1 \mathrm{H}), 3.04(\mathrm{dd}, J=14.7,9.9 \mathrm{~Hz}, 1 \mathrm{H}), 2.91(\mathrm{dd}, J=5.7,2.1 \mathrm{~Hz}$, 1H), $2.81(\mathrm{dd}, J=15.3,7.0 \mathrm{~Hz}, 1 \mathrm{H}), 2.70(\mathrm{dd}, J=7.6,2.0 \mathrm{~Hz}, 1 \mathrm{H}), 2.68(\mathrm{~m}, 1 \mathrm{H}), 2.24(\mathrm{~s}, 3 \mathrm{H})$, $2.15(\mathrm{dd}, J=13.8,4.8 \mathrm{~Hz}, 1 \mathrm{H}), 2.01(\mathrm{~m}, 1 \mathrm{H}), 1.76(\mathrm{dd}, J=13.9,8.4 \mathrm{~Hz}, 1 \mathrm{H}), 1.51-1.19(\mathrm{~m}, 5 \mathrm{H})$, $1.02(\mathrm{~d}, J=6.7 \mathrm{~Hz}, 3 \mathrm{H}), 0.93(\mathrm{~d}, J=6.8 \mathrm{~Hz}, 3 \mathrm{H}), 0.89(\mathrm{t}, J=7.2 \mathrm{~Hz}, 3 \mathrm{H})$, exchangeable protons not listed; ${ }^{13} \mathrm{C}$ NMR $\left(125 \mathrm{MHz}, \mathrm{CDCl}_{3}\right) \delta 165.9,152.9,147.1,145.0,144.7,136.0,134.7,131.5$, 130.4, 118.3, 115.1, 114.7, 112.9, 76.1, 74.9, 73.5, 72.5, 70.8, 62.5, 57.8, 39.9, 38.4, 38.2, 36.6, 35.4, 33.3, 20.0, 16.2, 15.8, 14.2, 13.9; IR (neat) 3274 (br), 2829, 1719, 1610, 1231, $1144 \mathrm{~cm}^{-1}$; ESIMS $m / z 553.4[\mathrm{M}+\mathrm{Na}]^{+}$; optical rotation $[\alpha]^{26}{ }_{\mathrm{D}}+165^{\circ}\left(c 0.050, \mathrm{CH}_{2} \mathrm{Cl}_{2}\right)$.

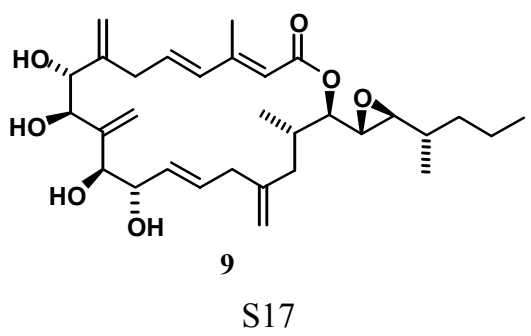


Data for 9: $\mathrm{R}_{f}=0.37(\mathrm{EtOAc}) ;{ }^{1} \mathrm{H} \mathrm{NMR}\left(500 \mathrm{MHz}, \mathrm{CDCl}_{3}\right) \delta 6.20(\mathrm{~d}, J=15.8 \mathrm{~Hz}, 1 \mathrm{H})$, 6.08 (ddd, $J=14.5,8.4,5.6 \mathrm{~Hz}, 1 \mathrm{H}), 5.79$ (s, 1H), 5.68 (dddd, $J=15.0,8.3,7.1,1.3 \mathrm{~Hz}, 1 \mathrm{H})$, $5.51(\mathrm{~s}, 1 \mathrm{H}), 5.43(\mathrm{~s}, 1 \mathrm{H}), 5.42(\mathrm{dd}, J=15.4,5.4 \mathrm{~Hz}, 1 \mathrm{H}), 5.37(\mathrm{~s}, 1 \mathrm{H}), 5.24(\mathrm{~s}, 1 \mathrm{H}), 4.84(\mathrm{~s}, 1 \mathrm{H})$, $4.76(\mathrm{~s}, 1 \mathrm{H}), 4.62(\mathrm{dd}, J=6.7,3.5 \mathrm{~Hz}, 1 \mathrm{H}), 4.42(\mathrm{~d}, J=3.2 \mathrm{~Hz}, 1 \mathrm{H}), 4.28(\mathrm{~d}, J=3.2 \mathrm{~Hz}, 1 \mathrm{H})$, $4.17(\mathrm{t}, J=4.5 \mathrm{~Hz}, 1 \mathrm{H}), 4.02(\mathrm{~d}, J=3.9 \mathrm{~Hz}, 1 \mathrm{H}), 3.22(\mathrm{dd}, J=14.5,5.4 \mathrm{~Hz}, 1 \mathrm{H}), 2.94(\mathrm{dd}, J=$ 13.1, 8.9 Hz, 1H), $2.92(\mathrm{dd}, J=7.0,2.3 \mathrm{~Hz}, 1 \mathrm{H}), 2.74(\mathrm{dd}, J=14.6,5.1 \mathrm{~Hz}, 1 \mathrm{H}), 2.68(\mathrm{dd}, J=$ 7.6, 2.2 Hz, 1H), 2.67 (dd, $J=14.4,7.7 \mathrm{~Hz}, 1 \mathrm{H}), 2.50(\mathrm{dd}, J=13.3,3.4 \mathrm{~Hz}, 1 \mathrm{H}), 2.26(\mathrm{~d}, J=1.1$ $\mathrm{Hz}, 3 \mathrm{H}), 2.10$ (m, 1H), $1.81(\mathrm{dd}, J=13.4,10.6 \mathrm{~Hz}, 1 \mathrm{H}), 1.53-1.25(\mathrm{~m}, 5 \mathrm{H}), 0.98(\mathrm{~d}, J=6.6 \mathrm{~Hz}$, $3 \mathrm{H}), 0.97(\mathrm{~d}, J=6.7 \mathrm{~Hz}, 3 \mathrm{H}), 0.90(\mathrm{t}, J=7.2 \mathrm{~Hz}, 3 \mathrm{H})$, exchangeable protons not listed; ${ }^{13} \mathrm{C}$ NMR $\left(125 \mathrm{MHz}, \mathrm{CDCl}_{3}\right) \delta 165.7,152.3,145.8,145.4,144.6,135.5,135.1,131.1,130.6,118.7,117.1$, $115.7,113.3,77.2,76.6,73.5,72.5,70.9,61.3,54.5,39.0,38.4,36.7,36.5,35.5,33.4,20.0$, 16.02, 15.96, 14.2, 14.1; IR (neat) 3414 (br), 2961, 1710, 1612, $1150 \mathrm{~cm}^{-1}$; ESIMS m/z 553.5 [M $+\mathrm{Na}]^{+}$; optical rotation $[\alpha]^{27}-116^{\circ}\left(c 0.020, \mathrm{CH}_{2} \mathrm{Cl}_{2}\right)$.

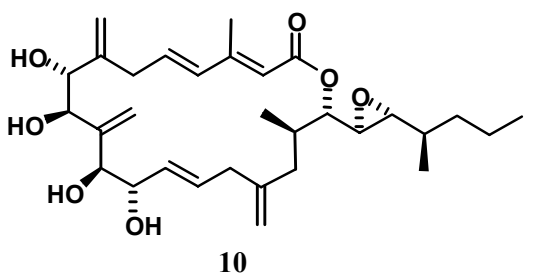

Data for 10: $\mathrm{R}_{f}=0.38\left(\right.$ EtOAc); ${ }^{1} \mathrm{H}$ NMR $\left(500 \mathrm{MHz}, \mathrm{CDCl}_{3}\right) \delta 6.27(\mathrm{~d}, J=15.5 \mathrm{~Hz}, 1 \mathrm{H})$, 6.08 (ddd, $J=15.1,10.0,5.0 \mathrm{~Hz}, 1 \mathrm{H}), 5.85$ (s, 1H), 5.68 (dddd, $J=15.4,7.1,7.1,1.2 \mathrm{~Hz}, 1 \mathrm{H}$ ), $5.49(\mathrm{dd}, J=15.4,5.0 \mathrm{~Hz}, 1 \mathrm{H}), 5.45(\mathrm{~s}, 1 \mathrm{H}), 5.34$ (s, 2H), $5.19(\mathrm{~s}, 1 \mathrm{H}), 4.85(\mathrm{~s}, 1 \mathrm{H}), 4.77(\mathrm{~s}, 1 \mathrm{H})$, 4.65 (dd, $J=6.7,3.7 \mathrm{~Hz}, 1 \mathrm{H}), 4.62$ (s br, 1H), 4.41 (s br, 1H), 4.20 (s br, 1H), 4.04 (s br, 1H), $3.20(\mathrm{dd}, J=14.8,4.4 \mathrm{~Hz}, 1 \mathrm{H}), 3.10(\mathrm{dd}, J=14.9,10.0,1 \mathrm{H}), 2.92(\mathrm{dd}, J=6.7,2.2 \mathrm{~Hz}, 1 \mathrm{H})$, 2.75-2.72 (m, 2H), $2.68(\mathrm{dd}, J=7.5,2.2 \mathrm{~Hz}, 1 \mathrm{H}), 2.51(\mathrm{dd}, J=13.7,3.4 \mathrm{~Hz}, 1 \mathrm{H}), 2.27(\mathrm{~d}, J=0.9$ $\mathrm{Hz}, 3 \mathrm{H}), 2.12(\mathrm{~m}, 1 \mathrm{H}), 1.85(\mathrm{dd}, J=14.0,10.5 \mathrm{~Hz}, 1 \mathrm{H}), 1.53-1.24(\mathrm{~m}, 5 \mathrm{H}), 1.00$ (d, $J=7.1 \mathrm{~Hz}$, $3 \mathrm{H}), 0.97(\mathrm{~d}, J=6.8 \mathrm{~Hz}, 3 \mathrm{H}), 0.90(\mathrm{t}, J=7.2 \mathrm{~Hz}, 3 \mathrm{H})$, exchangeable protons not listed; ${ }^{13} \mathrm{C}$ NMR $\left(125 \mathrm{MHz}, \mathrm{CDCl}_{3}\right) \delta 165.9,152.8,147.7,145.1,144.8,136.5,134.8,131.0,130.4,118.9,115.6$, $114.4,113.2,76.5,75.7,74.1,72.4,70.4,61.2,54.7,39.1,38.9,36.7,36.3,35.4,33.7,20.0,16.0$, 15.8, 14.2, 13.8; IR (neat) 3424 (br), 2928, 1709, 1611, $1163 \mathrm{~cm}^{-1}$; ESIMS m/z 553.4 [M + Na] $]^{+}$ optical rotation $[\alpha]^{27}+103^{\circ}\left(c 0.10, \mathrm{CH}_{2} \mathrm{Cl}_{2}\right)$. 


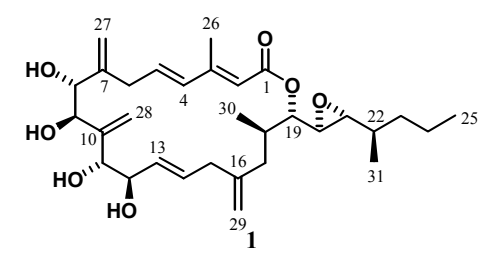

$\underline{\text { Isomer } 1}$

Natural Product

\begin{tabular}{|c|c|c|c|c|c|c|}
\hline Proton \# & $\delta_{\mathrm{H}}$ & Mult. & $J$ & $\delta_{\mathrm{H}}$ & Mult. & $J$ \\
\hline 2 & 5.80 & $\mathrm{~s}$ & - & 5.80 & $\mathrm{~s}$ & - \\
\hline 4 & 6.20 & $\mathrm{~d}$ & 15.5 & 6.27 & $\mathrm{~d}$ & 15.6 \\
\hline 5 & 6.09 & ddd & $15.5,10.3,4.5$ & 6.09 & ddd & $15.6,8.8,4.8$ \\
\hline $6 a$ & 3.22 & $\mathrm{dd}$ & $14.9,4.5$ & 3.20 & $\mathrm{dd}$ & $14.6,4.8$ \\
\hline $6 b$ & 3.12 & $\mathrm{dd}$ & $14.9,10.3$ & 3.13 & $\mathrm{dd}$ & $14.6,8.8$ \\
\hline 8 & 4.23 & $\mathrm{~s}$ br & - & 4.42 & $\mathrm{~s}$ br & - \\
\hline 9 & 4.29 & $\mathrm{~s}$ br & - & 4.58 & $\mathrm{~s}$ br & - \\
\hline 11 & 3.84 & $\mathrm{~s}$ br & - & 4.09 & $\mathrm{~s}$ br & - \\
\hline 12 & 4.21 & $\mathrm{~s} b r$ & - & 4.22 & $\mathrm{~s} b r$ & - \\
\hline 13 & 5.28 & $\mathrm{dd}$ & $15.3,2.6$ & 5.51 & dd & $15.4,4.1$ \\
\hline 14 & $5.78-5.73$ & $\mathrm{~m}$ & & 5.69 & $\mathrm{dt}$ & $15.4,7.7,7.7$ \\
\hline $15 \mathrm{a}$ & 2.77 & $\mathrm{dd}$ & $13.9,3.6$ & 2.76 & $\mathrm{~s}$ br $(2 \mathrm{H})$ & \\
\hline $15 \mathrm{~b}$ & 2.67 & $\mathrm{dd}$ & $13.9,10.3$ & 2.76 & $\mathrm{~s}$ br $(2 \mathrm{H})$ & \\
\hline $17 \mathrm{a}$ & 2.53 & $\mathrm{dd}$ & $12.9,3.1$ & 2.35 & $\mathrm{dd}$ & $14.0,5.5$ \\
\hline $17 \mathrm{~b}$ & 1.78 & $\mathrm{dd}$ & $12.9,10.8$ & 1.92 & $\mathrm{dd}$ & $14.0,9.5$ \\
\hline 18 & $2.18-2.13$ & $\mathrm{~m}$ & & 2.17 & $\mathrm{~m}$ & \\
\hline 19 & 4.55 & $\mathrm{dd}$ & $7.1,3.8$ & 4.72 & $\mathrm{dd}$ & $5.9,3.4$ \\
\hline 20 & 2.92 & $\mathrm{dd}$ & $7.1,2.2$ & 2.85 & $\mathrm{dd}$ & $5.9,1.9$ \\
\hline 21 & 2.70 & $\mathrm{dd}$ & $7.5,2.2$ & 2.76 & $\mathrm{dd}$ & 1.9 \\
\hline 22 & $1.70-1.26$ & $m(5 \mathrm{H})$ & & 1.38 & $\mathrm{~m}$ & \\
\hline 23 & $1.70-1.26$ & $m(5 \mathrm{H})$ & & $1.55-1.25$ & $\mathrm{~m}(4 \mathrm{H})$ & \\
\hline 24 & $1.70-1.26$ & $m(5 \mathrm{H})$ & & $1.55-1.25$ & $\mathrm{~m}(4 \mathrm{H})$ & \\
\hline $25(3 \mathrm{H})$ & 0.92 & $\mathrm{t}$ & 7.1 & 0.91 & $\mathrm{t}$ & 6.8 \\
\hline $26(3 \mathrm{H})$ & 2.30 & $\mathrm{~s}$ & - & 2.27 & $\mathrm{~s}$ & - \\
\hline $27 \mathrm{a}$ & 5.45 & $\mathrm{~s}$ & - & 5.36 & $\mathrm{~s}$ & - \\
\hline $27 b$ & 5.23 & s & - & 5.21 & $\mathrm{~s}$ & - \\
\hline $28 \mathrm{a}$ & 5.59 & $\mathrm{~s}$ & - & 5.49 & $\mathrm{~s}$ & - \\
\hline $28 b$ & 5.58 & $\mathrm{~s}$ & - & 5.37 & $\mathrm{~s}$ & - \\
\hline $29 a$ & 4.87 & $\mathrm{~s}$ & - & 4.88 & $\mathrm{~s}$ & - \\
\hline $29 b$ & 4.78 & $\mathrm{~s}$ & - & 4.79 & $\mathrm{~s}$ & - \\
\hline $30(3 \mathrm{H})$ & 0.99 & $\mathrm{~d}$ & 7.1 & 1.06 & $\mathrm{~d}$ & 7.0 \\
\hline $31(3 \mathrm{H})$ & 0.98 & $\mathrm{~d}$ & 6.8 & 0.93 & $\mathrm{~d}$ & 6.0 \\
\hline
\end{tabular}

Figure 1. ${ }^{1} \mathrm{H}$ NMR data for 1 in $\mathrm{CDCl}_{3}$ 


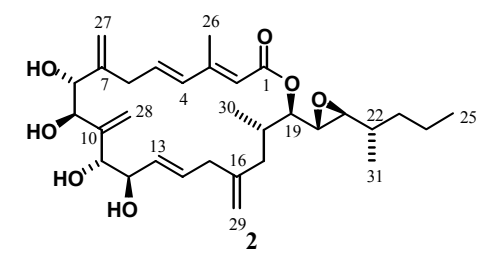

$\underline{\text { Isomer } 2}$

Natural Product

\begin{tabular}{|c|c|c|c|c|c|c|}
\hline Proton \# & $\delta_{\mathrm{H}}$ & Mult. & $J$ & $\delta_{\mathrm{H}}$ & Mult. & $J$ \\
\hline 2 & 5.80 & s & - & 5.80 & $\mathrm{~s}$ & - \\
\hline 4 & 6.14 & $\mathrm{~d}$ & 15.6 & 6.27 & $\mathrm{~d}$ & 15.6 \\
\hline 5 & 6.06 & $\mathrm{dt}$ & $15.4,6.7,6.7$ & 6.09 & ddd & $15.6,8.8,4.8$ \\
\hline $6 a$ & 3.19 & $\mathrm{dd}$ & $14.4,6.7$ & 3.20 & dd & $14.6,4.8$ \\
\hline $6 \mathrm{~b}$ & 2.96 & $\mathrm{dd}$ & $14.1,7.2$ & 3.13 & $\mathrm{dd}$ & $14.6,8.8$ \\
\hline 8 & 4.09 & $\mathrm{~d}$ & 4.6 & 4.42 & $\mathrm{~s}$ br & - \\
\hline 9 & 4.37 & $\mathrm{~d}$ & 4.6 & 4.58 & $\mathrm{~s}$ br & - \\
\hline 11 & 3.88 & $\mathrm{~d}$ & 3.3 & 4.09 & $\mathrm{~s}$ br & - \\
\hline 12 & 4.16 & $\mathrm{t}$ & $4.4,4.4$ & 4.22 & $\mathrm{~s} b r$ & - \\
\hline 13 & 5.36 & $\mathrm{dd}$ & $15.4,4.6$ & 5.51 & $\mathrm{dd}$ & $15.4,4.1$ \\
\hline 14 & 5.67 & $\mathrm{dt}$ & $14.1,6.4,6.4$ & 5.69 & $\mathrm{dt}$ & $15.4,7.7,7.7$ \\
\hline $15 \mathrm{a}$ & 2.71 & $\mathrm{dd}$ & $14.1,6.7$ & 2.76 & $\mathrm{~s}$ br $(2 \mathrm{H})$ & \\
\hline $15 b$ & 2.61 & $\mathrm{dd}$ & $14.1,8.0$ & 2.76 & $\mathrm{~s}$ br $(2 \mathrm{H})$ & \\
\hline $17 \mathrm{a}$ & 2.41 & $\mathrm{dd}$ & $14.1,3.6$ & 2.35 & $\mathrm{dd}$ & $14.0,5.5$ \\
\hline 19 & 4.58 & $\mathrm{dd}$ & $6.9,3.3$ & 4.72 & $\mathrm{dd}$ & $5.9,3.4$ \\
\hline 20 & 2.90 & $\mathrm{dd}$ & $6.7,3.1$ & 2.85 & $\mathrm{dd}$ & $5.9,1.9$ \\
\hline 21 & 2.66 & $\mathrm{dd}$ & $7.7,2.1$ & 2.76 & $\mathrm{dd}$ & 1.9 \\
\hline $26(3 \mathrm{H})$ & 2.23 & $\mathrm{~s}$ & - & 2.27 & $\mathrm{~s}$ & - \\
\hline $27 \mathrm{a}$ & 5.30 & $\mathrm{~s}$ & - & 5.36 & $\mathrm{~s}$ & - \\
\hline $27 \mathrm{~b}$ & 5.18 & $\mathrm{~s}$ & - & 5.21 & $\mathrm{~s}$ & - \\
\hline $28 \mathrm{a}$ & 5.46 & $\mathrm{~s}$ & - & 5.49 & $\mathrm{~s}$ & - \\
\hline $28 \mathrm{~b}$ & 5.37 & $\mathrm{~s}$ & - & 5.37 & $\mathrm{~s}$ & - \\
\hline $29 a$ & 4.83 & $\mathrm{~s}$ & - & 4.88 & $\mathrm{~s}$ & - \\
\hline $29 b$ & 4.75 & $\mathrm{~s}$ & - & 4.79 & $\mathrm{~s}$ & - \\
\hline
\end{tabular}

Figure 2. ${ }^{1} \mathrm{H}$ NMR data for $\mathbf{2}$ in $\mathrm{CDCl}_{3}$ 


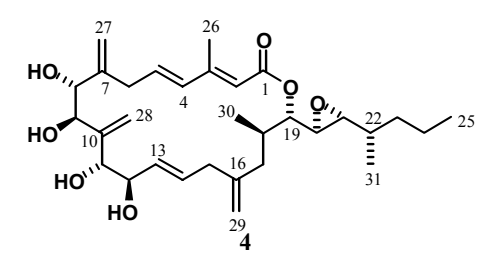

Isomer 4

$\underline{\text { Natural Product }}$

\begin{tabular}{|c|c|c|c|c|c|c|}
\hline Proton \# & $\delta_{\mathrm{H}}$ & Mult. & $J$ & $\delta_{\mathrm{H}}$ & Mult. & $J$ \\
\hline 2 & 5.79 & $\mathrm{~s}$ & - & 5.80 & $\mathrm{~s}$ & - \\
\hline 4 & 6.19 & $\mathrm{~d}$ & 15.6 & 6.27 & $\mathrm{~d}$ & 15.6 \\
\hline 5 & 6.08 & ddd & $14.9,9.8,3.8$ & 6.09 & ddd & $15.6,8.8,4.8$ \\
\hline $6 a$ & 3.22 & $\mathrm{dd}$ & $15.1,4.4$ & 3.20 & $\mathrm{dd}$ & $14.6,4.8$ \\
\hline $6 \mathrm{~b}$ & 3.11 & $\mathrm{dd}$ & $15.3,10.4$ & 3.13 & $\mathrm{dd}$ & $14.6,8.8$ \\
\hline 8 & 4.23 & $\mathrm{~s}$ br & - & 4.42 & $\mathrm{~s}$ br & - \\
\hline 9 & 4.28 & $\mathrm{~s}$ br & - & 4.58 & $\mathrm{~s}$ br & - \\
\hline 11 & 3.83 & $\mathrm{~s}$ br & - & 4.09 & $\mathrm{~s}$ br & - \\
\hline 12 & 4.20 & $\mathrm{~s} b r$ & - & 4.22 & $\mathrm{~s}$ br & - \\
\hline 13 & 5.26 & $\mathrm{~d}$ br & 16.2 & 5.51 & dd & $15.4,4.1$ \\
\hline 14 & $5.77-5.72$ & $\mathrm{~m}$ & & 5.69 & $\mathrm{dt}$ & $15.4,7.7,7.7$ \\
\hline $15 \mathrm{a}$ & 2.76 & $\mathrm{dd}$ & $13.6,4.0$ & 2.76 & $\mathrm{~s}$ br $(2 \mathrm{H})$ & \\
\hline $15 b$ & 2.66 & $\mathrm{dd}$ & $14.2,10.4$ & 2.76 & $\mathrm{~s}$ br $(2 \mathrm{H})$ & \\
\hline $17 \mathrm{a}$ & 2.51 & $\mathrm{dd}$ & $12.5,2.5$ & 2.35 & $\mathrm{dd}$ & $14.0,5.5$ \\
\hline $17 b$ & 1.79 & dd & $12.7,11.6$ & 1.92 & dd & $14.0,9.5$ \\
\hline 18 & 2.13 & $\mathrm{~m}$ & & 2.17 & $\mathrm{~m}$ & \\
\hline 19 & 4.61 & $\mathrm{dd}$ & $6.2,3.8$ & 4.72 & $\mathrm{dd}$ & $5.9,3.4$ \\
\hline 20 & 2.97 & $\mathrm{dd}$ & $6.1,2.1$ & 2.85 & $\mathrm{dd}$ & $5.9,1.9$ \\
\hline 21 & 2.69 & dd & $8.9,2.1$ & 2.76 & $\mathrm{dd}$ & 1.9 \\
\hline 22 & $1.44-1.22$ & $\mathrm{~m}(5 \mathrm{H})$ & & 1.38 & $\mathrm{~m}$ & \\
\hline 23 & $1.44-1.22$ & $\mathrm{~m}(5 \mathrm{H})$ & & $1.55-1.25$ & $m(4 \mathrm{H})$ & \\
\hline 24 & $1.44-1.22$ & $\mathrm{~m}(5 \mathrm{H})$ & & $1.55-1.25$ & $\mathrm{~m}(4 \mathrm{H})$ & \\
\hline $25(3 \mathrm{H})$ & 0.91 & $\mathrm{t}$ & 7.0 & 0.91 & $\mathrm{t}$ & 6.8 \\
\hline $26(3 \mathrm{H})$ & 2.29 & $\mathrm{~s}$ & - & 2.27 & $\mathrm{~s}$ & - \\
\hline $27 \mathrm{a}$ & 5.37 & $\mathrm{~s}$ & - & 5.36 & $\mathrm{~s}$ & - \\
\hline $27 b$ & 5.22 & $\mathrm{~s}$ & - & 5.21 & $\mathrm{~s}$ & - \\
\hline $28 \mathrm{a}$ & 5.59 & $\mathrm{~s}$ & - & 5.49 & $\mathrm{~s}$ & - \\
\hline $28 b$ & 5.57 & $\mathrm{~s}$ & - & 5.37 & $\mathrm{~s}$ & - \\
\hline $29 a$ & 4.86 & $\mathrm{~s}$ & - & 4.88 & $\mathrm{~s}$ & - \\
\hline $29 b$ & 4.78 & $\mathrm{~s}$ & - & 4.79 & $\mathrm{~s}$ & - \\
\hline $30(3 \mathrm{H})$ & 0.99 & $\mathrm{~d}$ & 7.0 & 1.06 & $\mathrm{~d}$ & 7.0 \\
\hline $31(3 \mathrm{H})$ & 1.01 & $\mathrm{~d}$ & 6.4 & 0.93 & $\mathrm{~d}$ & 6.0 \\
\hline
\end{tabular}

Figure 3. ${ }^{1} \mathrm{H}$ NMR data for 4 in $\mathrm{CDCl}_{3}$ 


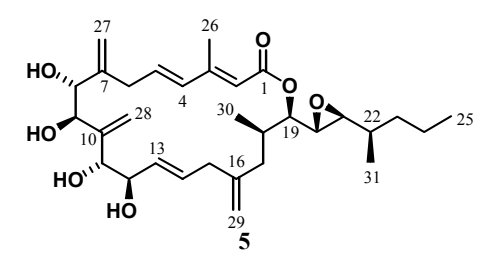

Isomer $\mathbf{5}$

$\underline{\text { Natural Product }}$

\begin{tabular}{|c|c|c|c|c|c|c|}
\hline Proton \# & $\delta_{\mathrm{H}}$ & Mult. & $J$ & $\delta_{\mathrm{H}}$ & Mult. & $J$ \\
\hline 2 & 5.81 & $\mathrm{~s}$ & - & 5.80 & s & - \\
\hline 4 & 6.19 & $\mathrm{~d}$ & 15.4 & 6.27 & $\mathrm{~d}$ & 15.6 \\
\hline 5 & 6.08 & ddd & $14.7,8.1,6.4$ & 6.09 & ddd & $15.6,8.8,4.8$ \\
\hline $6 a$ & 3.25 & dd & $14.4,6.4$ & 3.20 & dd & $14.6,4.8$ \\
\hline $6 b$ & 3.00 & dd & $14.4,8.2$ & 3.13 & dd & $14.6,8.8$ \\
\hline 8 & 4.32 & $\mathrm{~s}$ br & - & 4.42 & $\mathrm{~s}$ br & - \\
\hline 9 & 4.16 & $\mathrm{~s}$ br & - & 4.58 & $\mathrm{~s}$ br & - \\
\hline 11 & 3.95 & $\mathrm{~s}$ br & - & 4.09 & $\mathrm{~s}$ br & - \\
\hline 12 & 4.09 & $\mathrm{~m}$ & - & 4.22 & $\mathrm{~s}$ br & - \\
\hline 13 & 5.48 & ddm & $15.6,4.9$ & 5.51 & dd & $15.4,4.1$ \\
\hline 14 & 5.72 & dddd & $15.4,8.6,7.0,1.2$ & 5.69 & $\mathrm{dt}$ & $15.4,7.7,7.7$ \\
\hline $15 \mathrm{a}$ & $2.76-2.74$ & $\mathrm{~m}(2 \mathrm{H})$ & & 2.76 & $\mathrm{~s}$ br $(2 \mathrm{H})$ & \\
\hline $15 b$ & $2.76-2.74$ & $\mathrm{~m}(2 \mathrm{H})$ & & 2.76 & $\mathrm{~s}$ br $(2 \mathrm{H})$ & \\
\hline $17 \mathrm{a}$ & 2.17 & $\mathrm{dd}$ & $12.9,2.6$ & 2.35 & $\mathrm{dd}$ & $14.0,5.5$ \\
\hline $17 \mathrm{~b}$ & 1.65 & $\mathrm{dd}$ & $13.3,9.6$ & 1.92 & $\mathrm{dd}$ & $14.0,9.5$ \\
\hline 18 & 1.94 & $\mathrm{~m}$ & & 2.17 & $\mathrm{~m}$ & \\
\hline 19 & 4.60 & $\mathrm{dd}$ & $10.3,5.5$ & 4.72 & dd & $5.9,3.4$ \\
\hline 20 & 3.02 & $\mathrm{dd}$ & $5.6,2.2$ & 2.85 & $\mathrm{dd}$ & $5.9,1.9$ \\
\hline 21 & 2.72 & $\mathrm{dd}$ & $6.7,2.1$ & 2.76 & $\mathrm{dd}$ & 1.9 \\
\hline 22 & $1.47-1.21$ & $\mathrm{~m}(5 \mathrm{H})$ & & 1.38 & $\mathrm{~m}$ & \\
\hline 23 & $1.47-1.21$ & $\mathrm{~m}(5 \mathrm{H})$ & & $1.55-1.25$ & $\mathrm{~m}(4 \mathrm{H})$ & \\
\hline 24 & $1.47-1.21$ & $\mathrm{~m}(5 \mathrm{H})$ & & $1.55-1.25$ & $\mathrm{~m}(4 \mathrm{H})$ & \\
\hline $25(3 \mathrm{H})$ & 0.92 & $\mathrm{t}$ & 7.2 & 0.91 & $\mathrm{t}$ & 6.8 \\
\hline $26(3 \mathrm{H})$ & 2.26 & $\mathrm{~d}$ & 1.0 & 2.27 & $\mathrm{~s}$ & - \\
\hline $27 \mathrm{a}$ & 5.34 & $\mathrm{~s}$ & - & 5.36 & $\mathrm{~s}$ & - \\
\hline $27 b$ & 5.24 & $\mathrm{~s}$ & - & 5.21 & $\mathrm{~s}$ & - \\
\hline $28 \mathrm{a}$ & 5.49 & $\mathrm{~s}$ & - & 5.49 & $\mathrm{~s}$ & - \\
\hline $28 b$ & 5.44 & $\mathrm{~s}$ & - & 5.37 & $\mathrm{~s}$ & - \\
\hline $29 a$ & 4.83 & $\mathrm{~s}$ & - & 4.88 & $\mathrm{~s}$ & - \\
\hline $29 b$ & 4.75 & $\mathrm{~s}$ & - & 4.79 & s & - \\
\hline $30(3 \mathrm{H})$ & 1.04 & $\mathrm{~d}$ & 6.7 & 1.06 & $d$ & 7.0 \\
\hline $31(3 \mathrm{H})$ & 1.00 & d & 6.6 & 0.93 & d & 6.0 \\
\hline
\end{tabular}

Figure 4. ${ }^{1} \mathrm{H}$ NMR data for 5 in $\mathrm{CDCl}_{3}$ 


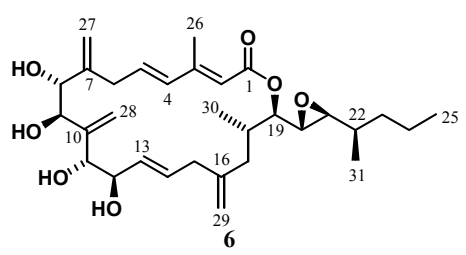

Isomer 6

Natural Product

\begin{tabular}{|c|c|c|c|c|c|c|}
\hline Proton \# & $\delta_{\mathrm{H}}$ & Mult. & $J$ & $\delta_{\mathrm{H}}$ & Mult. & $J$ \\
\hline 2 & 5.82 & s & - & 5.80 & $\mathrm{~s}$ & - \\
\hline 4 & 6.16 & $\mathrm{~d}$ & 15.9 & 6.27 & d & 15.6 \\
\hline 5 & 6.08 & ddd & $15.5,7.0,6.7$ & 6.09 & ddd & $15.6,8.8,4.8$ \\
\hline $6 a$ & 3.21 & $\mathrm{dd}$ & $14.7,6.5$ & 3.20 & dd & $14.6,4.8$ \\
\hline $6 b$ & 2.98 & $\mathrm{dd}$ & $14.5,7.4$ & 3.13 & $\mathrm{dd}$ & $14.6,8.8$ \\
\hline 8 & 4.38 & $\mathrm{~d}$ & 4.3 & 4.42 & $\mathrm{~s}$ br & - \\
\hline 9 & 4.10 & $\mathrm{~d}$ & 4.3 & 4.58 & $\mathrm{~s}$ br & - \\
\hline 11 & 3.89 & $\mathrm{~d}$ & 3.5 & 4.09 & $\mathrm{~s}$ br & - \\
\hline 12 & 4.17 & $\mathrm{dd}$ & $4.0,3.7$ & 4.22 & $\mathrm{~s}$ br & - \\
\hline 13 & 5.38 & $\mathrm{dd}$ & $15.4,5.4$ & 5.51 & dd & $15.4,4.1$ \\
\hline 14 & 5.68 & dddd & $15.4,7.8,6.6,1.2$ & 5.69 & $\mathrm{dt}$ & $15.4,7.7,7.7$ \\
\hline $15 \mathrm{a}$ & 2.73 & $\mathrm{dd}$ & $13.9,6.0$ & 2.76 & $\mathrm{~s}$ br $(2 \mathrm{H})$ & \\
\hline $15 b$ & 2.63 & dd & $14.7,8.3$ & 2.76 & $\mathrm{~s}$ br $(2 \mathrm{H})$ & \\
\hline $17 \mathrm{a}$ & 2.40 & dd & $13.7,3.9$ & 2.35 & $\mathrm{dd}$ & $14.0,5.5$ \\
\hline $17 b$ & 1.82 & $\mathrm{dd}$ & $13.7,10.1$ & 1.92 & $\mathrm{dd}$ & $14.0,9.5$ \\
\hline 18 & 2.11 & $\mathrm{~m}$ & & 2.17 & $\mathrm{~m}$ & \\
\hline 19 & 4.67 & $\mathrm{dd}$ & $6.1,3.4$ & 4.72 & $\mathrm{dd}$ & $5.9,3.4$ \\
\hline 20 & 2.97 & $\mathrm{dd}$ & $6.1,2.2$ & 2.85 & $\mathrm{dd}$ & $5.9,1.9$ \\
\hline 21 & 2.66 & $\mathrm{dd}$ & $7.0,2.2$ & 2.76 & dd & 1.9 \\
\hline 22 & $1.43-1.20$ & $\mathrm{~m}(5 \mathrm{H})$ & & 1.38 & $\mathrm{~m}$ & \\
\hline 23 & $1.43-1.20$ & $\mathrm{~m}(5 \mathrm{H})$ & & $1.55-1.25$ & m (4H) & \\
\hline 24 & $1.43-1.20$ & $\mathrm{~m}(5 \mathrm{H})$ & & $1.55-1.25$ & $\mathrm{~m}(4 \mathrm{H})$ & \\
\hline $25(3 \mathrm{H})$ & 0.91 & $\mathrm{t}$ & 7.0 & 0.91 & $\mathrm{t}$ & 6.8 \\
\hline $26(3 \mathrm{H})$ & 2.25 & $\mathrm{~d}$ & 1.1 & 2.27 & $\mathrm{~s}$ & - \\
\hline $27 \mathrm{a}$ & 5.32 & $\mathrm{~s}$ & - & 5.36 & s & - \\
\hline $27 b$ & 5.20 & $\mathrm{~s}$ & - & 5.21 & s & - \\
\hline $28 \mathrm{a}$ & 5.47 & s & - & 5.49 & $\mathrm{~s}$ & - \\
\hline $28 b$ & 5.39 & $\mathrm{~s}$ & - & 5.37 & s & - \\
\hline $29 a$ & 4.85 & $\mathrm{~s}$ & - & 4.88 & $\mathrm{~s}$ & - \\
\hline $29 b$ & 4.77 & s & - & 4.79 & s & - \\
\hline $30(3 \mathrm{H})$ & 1.01 & $\mathrm{~d}$ & 7.0 & 1.06 & $\mathrm{~d}$ & 7.0 \\
\hline $31(3 \mathrm{H})$ & 1.00 & $\mathrm{~d}$ & 6.5 & 0.93 & $\mathrm{~d}$ & 6.0 \\
\hline
\end{tabular}

Figure 5. ${ }^{\mathrm{I}} \mathrm{H}$ NMR data for $\mathbf{6}$ in $\mathrm{CDCl}_{3}$ 


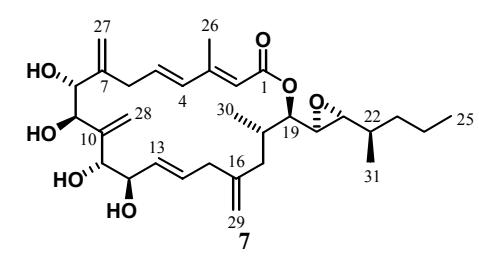

Isomer 7

$\underline{\text { Natural Product }}$

\begin{tabular}{|c|c|c|c|c|c|c|}
\hline Proton \# & $\delta_{\mathrm{H}}$ & Mult. & $J$ & $\delta_{\mathrm{H}}$ & Mult. & $J$ \\
\hline 2 & 5.77 & $\mathrm{~s}$ & - & 5.80 & $\mathrm{~s}$ & - \\
\hline 4 & 6.16 & $\mathrm{~d}$ & 15.6 & 6.27 & $\mathrm{~d}$ & 15.6 \\
\hline 5 & 6.08 & ddd & $15.9,6.8,6.5$ & 6.09 & ddd & $15.6,8.8,4.8$ \\
\hline $6 a$ & 3.21 & $\mathrm{dd}$ & $14.9,7.0$ & 3.20 & $\mathrm{dd}$ & $14.6,4.8$ \\
\hline $6 b$ & 2.98 & dd & $14.4,7.1$ & 3.13 & dd & $14.6,8.8$ \\
\hline 8 & 4.38 & $\mathrm{~s} b r$ & - & 4.42 & $\mathrm{~s}$ br & - \\
\hline 9 & 4.12 & $\mathrm{~s} b r$ & - & 4.58 & $\mathrm{~s} b r$ & - \\
\hline 11 & 3.91 & $\mathrm{~s}$ br & - & 4.09 & $\mathrm{~s}$ br & - \\
\hline 12 & 4.18 & $\mathrm{~s}$ br & - & 4.22 & s br & - \\
\hline 13 & 5.41 & $\mathrm{dd}$ & $15.5,5.4$ & 5.51 & $\mathrm{dd}$ & $15.4,4.1$ \\
\hline 14 & 5.69 & ddd & $14.2,6.6,6.3$ & 5.69 & $\mathrm{dt}$ & $15.4,7.7,7.7$ \\
\hline $15 \mathrm{a}$ & 2.74 & $\mathrm{dd}$ & $14.8,6.7$ & 2.76 & $\mathrm{~s}$ br $(2 \mathrm{H})$ & \\
\hline $15 b$ & 2.66 & $\mathrm{dd}$ & $14.8,7.3$ & 2.76 & $\mathrm{~s}$ br $(2 \mathrm{H})$ & \\
\hline $17 \mathrm{a}$ & 2.26 & $\mathrm{~d}$ br & 14.0 & 2.35 & $\mathrm{dd}$ & $14.0,5.5$ \\
\hline $17 \mathrm{~b}$ & 1.88 & $\mathrm{dd}$ & $13.9,9.2$ & 1.92 & $\mathrm{dd}$ & $14.0,9.5$ \\
\hline 18 & 2.14 & $\mathrm{~m}$ & & 2.17 & $\mathrm{~m}$ & \\
\hline 19 & 4.67 & $\mathrm{dd}$ & $6.4,3.2$ & 4.72 & $\mathrm{dd}$ & $5.9,3.4$ \\
\hline 20 & 2.84 & $\mathrm{dd}$ & $6.2,2.1$ & 2.85 & $\mathrm{dd}$ & $5.9,1.9$ \\
\hline 21 & 2.77 & $\mathrm{dd}$ & $7.6,2.2$ & 2.76 & dd & 1.9 \\
\hline 22 & $1.51-1.25$ & $m(5 \mathrm{H})$ & & 1.38 & $\mathrm{~m}$ & \\
\hline 23 & $1.51-1.25$ & $\mathrm{~m}(5 \mathrm{H})$ & & $1.55-1.25$ & $\mathrm{~m}(4 \mathrm{H})$ & \\
\hline 24 & $1.51-1.25$ & $\mathrm{~m}(5 \mathrm{H})$ & & $1.55-1.25$ & $\mathrm{~m}(4 \mathrm{H})$ & \\
\hline $25(3 \mathrm{H})$ & 0.91 & $\mathrm{t}$ & 7.0 & 0.91 & $\mathrm{t}$ & 6.8 \\
\hline $26(3 \mathrm{H})$ & 2.24 & $\mathrm{~d}$ & 1.0 & 2.27 & $\mathrm{~s}$ & - \\
\hline $27 \mathrm{a}$ & 5.33 & $\mathrm{~s}$ & - & 5.36 & $\mathrm{~s}$ & - \\
\hline $27 b$ & 5.21 & $\mathrm{~s}$ & - & 5.21 & $\mathrm{~s}$ & - \\
\hline $28 \mathrm{a}$ & 5.48 & $\mathrm{~s}$ & - & 5.49 & $\mathrm{~s}$ & - \\
\hline $28 b$ & 5.39 & $\mathrm{~s}$ & - & 5.37 & $\mathrm{~s}$ & - \\
\hline $29 a$ & 4.87 & $\mathrm{~s}$ & - & 4.88 & $\mathrm{~s}$ & - \\
\hline $29 b$ & 4.79 & $\mathrm{~s}$ & - & 4.79 & $\mathrm{~s}$ & - \\
\hline $30(3 \mathrm{H})$ & 1.05 & $\mathrm{~d}$ & 7.1 & 1.06 & $\mathrm{~d}$ & 7.0 \\
\hline $31(3 \mathrm{H})$ & 0.89 & $\mathrm{~d}$ & 7.1 & 0.93 & $\mathrm{~d}$ & 6.0 \\
\hline
\end{tabular}

Figure 6. ${ }^{1} \mathrm{H}$ NMR data for 7 in $\mathrm{CDCl}_{3}$ 


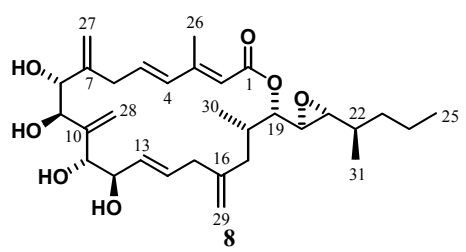

Isomer $\mathbf{8}$

Natural Product

\begin{tabular}{|c|c|c|c|c|c|c|}
\hline Proton \# & $\delta_{\mathrm{H}}$ & Mult. & $J$ & $\delta_{\mathrm{H}}$ & Mult. & $J$ \\
\hline 2 & 5.72 & $\mathrm{~s}$ & - & 5.80 & $\mathrm{~s}$ & - \\
\hline 4 & 6.16 & $\mathrm{~d}$ & 15.6 & 6.27 & d & 15.6 \\
\hline 5 & 6.03 & ddd & $14.9,9.8,4.8$ & 6.09 & ddd & $15.6,8.8,4.8$ \\
\hline $6 a$ & 3.16 & $\mathrm{dd}$ & $14.4,4.3$ & 3.20 & $\mathrm{dd}$ & $14.6,4.8$ \\
\hline $6 b$ & 3.04 & dd & $14.7,9.9$ & 3.13 & dd & $14.6,8.8$ \\
\hline 8 & 4.36 & $\mathrm{~s}$ br & - & 4.42 & $\mathrm{~s}$ br & - \\
\hline 9 & 4.30 & $\mathrm{~s}$ br & - & 4.58 & $\mathrm{~s}$ br & - \\
\hline 11 & 3.99 & $\mathrm{~s}$ br & - & 4.09 & $\mathrm{~s}$ br & - \\
\hline 12 & 4.27 & $\mathrm{~s}$ br & - & 4.22 & $\mathrm{~s}$ br & - \\
\hline 13 & 5.53 & dd & $15.6,5.4$ & 5.51 & dd & $15.4,4.1$ \\
\hline 14 & 5.75 & ddd & $15.3,7.3,6.4$ & 5.69 & $\mathrm{dt}$ & $15.4,7.7,7.7$ \\
\hline $15 \mathrm{a}$ & 2.81 & $\mathrm{dd}$ & $15.3,7.0$ & 2.76 & $\mathrm{~s}$ br $(2 \mathrm{H})$ & \\
\hline $15 b$ & 2.68 & $\mathrm{~m}$ & & 2.76 & $\mathrm{~s}$ br $(2 \mathrm{H})$ & \\
\hline $17 \mathrm{a}$ & 2.15 & dd & $13.8,4.8$ & 2.35 & dd & $14.0,5.5$ \\
\hline $17 \mathrm{~b}$ & 1.76 & $\mathrm{dd}$ & $13.9,8.4$ & 1.92 & $\mathrm{dd}$ & $14.0,9.5$ \\
\hline 18 & 2.01 & $\mathrm{~m}$ & & 2.17 & $\mathrm{~m}$ & \\
\hline 19 & 4.56 & $\mathrm{dd}$ & $10.3,5.6$ & 4.72 & $\mathrm{dd}$ & $5.9,3.4$ \\
\hline 20 & 2.91 & dd & $5.7,2.1$ & 2.85 & dd & $5.9,1.9$ \\
\hline 21 & 2.70 & $\mathrm{dd}$ & $7.6,2.0$ & 2.76 & dd & 1.9 \\
\hline 22 & $1.51-1.19$ & $\mathrm{~m}(5 \mathrm{H})$ & & 1.38 & $\mathrm{~m}$ & \\
\hline 23 & $1.51-1.19$ & $\mathrm{~m}(5 \mathrm{H})$ & & $1.55-1.25$ & $\mathrm{~m}(4 \mathrm{H})$ & \\
\hline 24 & $1.51-1.19$ & $\mathrm{~m}(5 \mathrm{H})$ & & $1.55-1.25$ & $\mathrm{~m}(4 \mathrm{H})$ & \\
\hline $25(3 \mathrm{H})$ & 0.89 & $\mathrm{t}$ & 7.2 & 0.91 & $\mathrm{t}$ & 6.8 \\
\hline $26(3 \mathrm{H})$ & 2.24 & $\mathrm{~s}$ & - & 2.27 & $\mathrm{~s}$ & - \\
\hline $27 \mathrm{a}$ & 5.33 & $\mathrm{~s}$ & - & 5.36 & $\mathrm{~s}$ & - \\
\hline $27 b$ & 5.20 & $\mathrm{~s}$ & - & 5.21 & $\mathrm{~s}$ & - \\
\hline $28 \mathrm{a}$ & 5.49 & $\mathrm{~s}$ & - & 5.49 & $\mathrm{~s}$ & - \\
\hline $28 b$ & 5.42 & $\mathrm{~s}$ & - & 5.37 & $\mathrm{~s}$ & - \\
\hline $29 a$ & 4.74 & $\mathrm{~s}$ & - & 4.88 & $\mathrm{~s}$ & - \\
\hline $29 b$ & 4.73 & $\mathrm{~S}$ & - & 4.79 & $\mathrm{~S}$ & - \\
\hline $30(3 \mathrm{H})$ & 1.02 & $\mathrm{~d}$ & 6.7 & 1.06 & $\mathrm{~d}$ & 7.0 \\
\hline $31(3 \mathrm{H})$ & 0.93 & $\mathrm{~d}$ & 6.8 & 0.93 & $\mathrm{~d}$ & 6.0 \\
\hline
\end{tabular}

Figure 7. ${ }^{\mathrm{I}} \mathrm{H}$ NMR data for 8 in $\mathrm{CDCl}_{3}$ 


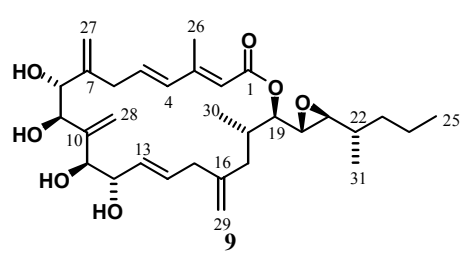

Isomer 9

$\underline{\text { Natural Product }}$

Proton \#

$\delta_{\mathrm{H}}$

Mult. J

$\delta_{\mathrm{H}}$

Mult. $J$

\begin{tabular}{|c|c|c|c|c|c|c|}
\hline 2 & 5.79 & $\mathrm{~s}$ & - & 5.80 & $\mathrm{~s}$ & - \\
\hline 4 & 6.20 & d & 15.8 & 6.27 & $\mathrm{~d}$ & 15.6 \\
\hline 5 & 6.08 & ddd & $14.5,8.4,5.6$ & 6.09 & ddd & $15.6,8.8,4.8$ \\
\hline $6 a$ & 3.22 & $\mathrm{dd}$ & $14.5,5.4$ & 3.20 & $\mathrm{dd}$ & $14.6,4.8$ \\
\hline $6 b$ & 2.94 & $\mathrm{dd}$ & $13.1,8.9$ & 3.13 & $\mathrm{dd}$ & $14.6,8.8$ \\
\hline 8 & 4.42 & d & 3.2 & 4.42 & $\mathrm{~s}$ br & - \\
\hline 9 & 4.28 & $\mathrm{~d}$ & 3.2 & 4.58 & $\mathrm{~s}$ br & - \\
\hline 11 & 4.02 & d & 3.9 & 4.09 & $\mathrm{~s}$ br & - \\
\hline 12 & 4.17 & $\mathrm{t}$ & $4.5,4.5$ & 4.22 & $\mathrm{~s}$ br & - \\
\hline 13 & 5.42 & $\mathrm{dd}$ & $15.4,5.4$ & 5.51 & $\mathrm{dd}$ & $15.4,4.1$ \\
\hline 14 & 5.68 & dddd & $15.0,8.3,7.1,1.3$ & 5.69 & $\mathrm{dt}$ & $15.4,7.7,7.7$ \\
\hline $15 \mathrm{a}$ & 2.74 & $\mathrm{dd}$ & $14.6,5.1$ & 2.76 & $\mathrm{~s}$ br $(2 \mathrm{H})$ & \\
\hline $15 b$ & 2.67 & $\mathrm{dd}$ & $14.4,7.7$ & 2.76 & $\mathrm{~s}$ br $(2 \mathrm{H})$ & \\
\hline $17 \mathrm{a}$ & 2.50 & $\mathrm{dd}$ & $13.3,3.4$ & 2.35 & $\mathrm{dd}$ & $14.0,5.5$ \\
\hline $17 \mathrm{~b}$ & 1.81 & $\mathrm{dd}$ & $13.4,10.6$ & 1.92 & $\mathrm{dd}$ & $14.0,9.5$ \\
\hline 18 & 2.10 & $\mathrm{~m}$ & & 2.17 & $\mathrm{~m}$ & \\
\hline 19 & 4.62 & $\mathrm{dd}$ & $6.7,3.5$ & 4.72 & $\mathrm{dd}$ & $5.9,3.4$ \\
\hline 20 & 2.92 & $\mathrm{dd}$ & $7.0,2.3$ & 2.85 & $\mathrm{dd}$ & $5.9,1.9$ \\
\hline 21 & 2.68 & $\mathrm{dd}$ & $7.6,2.2$ & 2.76 & $\mathrm{dd}$ & 1.9 \\
\hline 22 & $1.53-1.25$ & $\mathrm{~m}(5 \mathrm{H})$ & & 1.38 & $\mathrm{~m}$ & \\
\hline 23 & $1.53-1.25$ & $\mathrm{~m}(5 \mathrm{H})$ & & $1.55-1.25$ & $\mathrm{~m}(4 \mathrm{H})$ & \\
\hline 24 & $1.53-1.25$ & $\mathrm{~m}(5 \mathrm{H})$ & & $1.55-1.25$ & $\mathrm{~m}(4 \mathrm{H})$ & \\
\hline $25(3 \mathrm{H})$ & 0.90 & $\mathrm{t}$ & 7.2 & 0.91 & $\mathrm{t}$ & 6.8 \\
\hline $26(3 \mathrm{H})$ & 2.26 & $\mathrm{~d}$ & 1.1 & 2.27 & $\mathrm{~s}$ & - \\
\hline $27 \mathrm{a}$ & 5.37 & $\mathrm{~s}$ & - & 5.36 & $\mathrm{~s}$ & - \\
\hline $27 \mathrm{~b}$ & 5.24 & $\mathrm{~s}$ & - & 5.21 & $\mathrm{~s}$ & - \\
\hline $28 \mathrm{a}$ & 5.51 & $\mathrm{~s}$ & - & 5.49 & $\mathrm{~s}$ & - \\
\hline $28 \mathrm{~b}$ & 5.43 & $\mathrm{~s}$ & - & 5.37 & $\mathrm{~s}$ & - \\
\hline $29 a$ & 4.84 & $\mathrm{~s}$ & - & 4.88 & $\mathrm{~s}$ & - \\
\hline $29 b$ & 4.76 & $\mathrm{~s}$ & - & 4.79 & $\mathrm{~s}$ & - \\
\hline $30(3 \mathrm{H})$ & 0.98 & $\mathrm{~d}$ & 6.6 & 1.06 & $\mathrm{~d}$ & 7.0 \\
\hline $31(3 \mathrm{H})$ & 0.97 & $\mathrm{~d}$ & 6.7 & 0.93 & $\mathrm{~d}$ & 6.0 \\
\hline
\end{tabular}

Figure 8. ${ }^{\mathrm{I}} \mathrm{H}$ NMR data for 9 in $\mathrm{CDCl}_{3}$ 


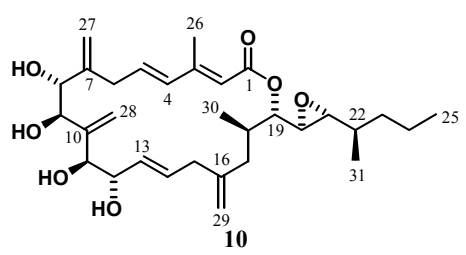

Isomer 10

Natural Product

\begin{tabular}{|c|c|c|c|c|c|c|}
\hline Proton \# & $\delta_{\mathrm{H}}$ & Mult. & $J$ & $\delta_{\mathrm{H}}$ & Mult. & $J$ \\
\hline 2 & 5.85 & s & - & 5.80 & s & - \\
\hline 4 & 6.27 & $\mathrm{~d}$ & 15.5 & 6.27 & $\mathrm{~d}$ & 15.6 \\
\hline 5 & 6.08 & ddd & $15.1,10.0,5.0$ & 6.09 & ddd & $15.6,8.8,4.8$ \\
\hline $6 a$ & 3.20 & $\mathrm{dd}$ & $14.8,4.4$ & 3.20 & $\mathrm{dd}$ & $14.6,4.8$ \\
\hline $6 b$ & 3.10 & $\mathrm{dd}$ & $14.9,10.0$ & 3.13 & $\mathrm{dd}$ & $14.6,8.8$ \\
\hline 8 & 4.41 & $\mathrm{~s}$ br & & 4.42 & $\mathrm{~s}$ br & - \\
\hline 9 & 4.62 & $\mathrm{~s}$ br & & 4.58 & $\mathrm{~s}$ br & - \\
\hline 11 & 4.04 & $\mathrm{~s}$ br & & 4.09 & $\mathrm{~s}$ br & - \\
\hline 12 & 4.20 & $\mathrm{~s}$ br & & 4.22 & $\mathrm{~s}$ br & - \\
\hline 13 & 5.49 & dd & $15.4,5.0$ & 5.51 & $\mathrm{dd}$ & $15.4,4.1$ \\
\hline 14 & 5.68 & dddd & $15.4,7.1,7.1,1.2$ & 5.69 & $\mathrm{dt}$ & $15.4,7.7,7.7$ \\
\hline $15 \mathrm{a}$ & $2.75-2.72$ & $\mathrm{~m}(2 \mathrm{H})$ & & 2.76 & $\mathrm{~s}$ br $(2 \mathrm{H})$ & \\
\hline $15 b$ & $2.75-2.72$ & $\mathrm{~m}(2 \mathrm{H})$ & & 2.76 & $\mathrm{~s}$ br $(2 \mathrm{H})$ & \\
\hline $17 \mathrm{a}$ & 2.51 & $\mathrm{dd}$ & $13.7,3.4$ & 2.35 & dd & $14.0,5.5$ \\
\hline $17 \mathrm{~b}$ & 1.85 & dd & $14.0,10.5$ & 1.92 & $\mathrm{dd}$ & $14.0,9.5$ \\
\hline 18 & 2.12 & $\mathrm{~m}$ & & 2.17 & $\mathrm{~m}$ & \\
\hline 19 & 4.65 & $\mathrm{dd}$ & $6.7,3.7$ & 4.72 & $\mathrm{dd}$ & $5.9,3.4$ \\
\hline 20 & 2.92 & $\mathrm{dd}$ & $6.7,2.2$ & 2.85 & $\mathrm{dd}$ & $5.9,1.9$ \\
\hline 21 & 2.68 & $\mathrm{dd}$ & $7.5,2.2$ & 2.76 & dd & 1.9 \\
\hline 22 & $1.53-1.24$ & $\mathrm{~m}(5 \mathrm{H})$ & & 1.38 & $\mathrm{~m}$ & \\
\hline 23 & $1.53-1.24$ & $\mathrm{~m}(5 \mathrm{H})$ & & $1.55-1.25$ & m (4H) & \\
\hline 24 & $1.53-1.24$ & $\mathrm{~m}(5 \mathrm{H})$ & & $1.55-1.25$ & $\mathrm{~m}(4 \mathrm{H})$ & \\
\hline $25(3 \mathrm{H})$ & 0.90 & $\mathrm{t}$ & 7.2 & 0.91 & $\mathrm{t}$ & 6.8 \\
\hline $26(3 \mathrm{H})$ & 2.27 & $\mathrm{~d}$ & 0.9 & 2.27 & $\mathrm{~s}$ & - \\
\hline $27 \mathrm{a}$ & 5.34 & s & - & 5.36 & s & - \\
\hline $27 b$ & 5.19 & s & - & 5.21 & s & - \\
\hline $28 \mathrm{a}$ & 5.45 & s & - & 5.49 & s & - \\
\hline $28 b$ & 5.34 & s & - & 5.37 & $\mathrm{~s}$ & - \\
\hline $29 a$ & 4.85 & s & - & 4.88 & s & - \\
\hline $29 b$ & 4.77 & s & - & 4.79 & s & - \\
\hline $30(3 \mathrm{H})$ & 1.00 & $\mathrm{~d}$ & 7.1 & 1.06 & $\mathrm{~d}$ & 7.0 \\
\hline $31(3 \mathrm{H})$ & 0.97 & $\mathrm{~d}$ & 6.8 & 0.93 & $\mathrm{~d}$ & 6.0 \\
\hline
\end{tabular}

Figure 9. ${ }^{1} \mathrm{H}$ NMR data for $\mathbf{1 0}$ in $\mathrm{CDCl}_{3}$ 


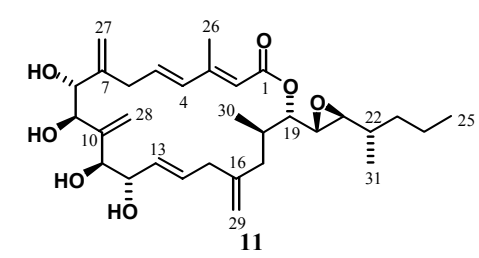

Isomer 11

$\underline{\text { Natural Product }}$

\begin{tabular}{|c|c|c|c|c|c|c|}
\hline Proton \# & $\delta_{\mathrm{H}}$ & Mult. & $J$ & $\delta_{\mathrm{H}}$ & Mult. & $J$ \\
\hline 2 & 5.80 & $\mathrm{~s}$ & - & 5.80 & s & - \\
\hline 4 & 6.28 & $\mathrm{~d}$ & 15.4 & 6.27 & $\mathrm{~d}$ & 15.6 \\
\hline 5 & 6.10 & ddd & $15.5,9.6,5.1$ & 6.09 & ddd & $15.6,8.8,4.8$ \\
\hline $6 a$ & 3.21 & $\mathrm{dd}$ & $14.9,5.0$ & 3.20 & $\mathrm{dd}$ & $14.6,4.8$ \\
\hline $6 \mathrm{~b}$ & 3.11 & dd & $14.7,9.3$ & 3.13 & dd & $14.6,8.8$ \\
\hline 8 & 4.43 & $\mathrm{~s}$ br & - & 4.42 & $\mathrm{~s}$ br & - \\
\hline 9 & 4.59 & $\mathrm{~s}$ br & - & 4.58 & $\mathrm{~s}$ br & - \\
\hline 11 & 4.06 & $\mathrm{tbr}$ & 3.8 & 4.09 & $\mathrm{~s}$ br & - \\
\hline 12 & 4.22 & $\mathrm{~s}$ br & - & 4.22 & $\mathrm{~s}$ br & - \\
\hline 13 & 5.50 & $\mathrm{dd}$ & $15.4,4.8$ & 5.51 & $\mathrm{dd}$ & $15.4,4.1$ \\
\hline 14 & 5.69 & dddd & $15.3,7.3,7.3,1.5$ & 5.69 & $\mathrm{dt}$ & $15.4,7.7,7.7$ \\
\hline $15 \mathrm{a}$ & 2.76 & $\mathrm{~s}$ br $(2 \mathrm{H})$ & & 2.76 & $\mathrm{~s}$ br $(2 \mathrm{H})$ & \\
\hline $15 b$ & 2.76 & $\mathrm{~s}$ br $(2 \mathrm{H})$ & & 2.76 & $\mathrm{~s}$ br $(2 \mathrm{H})$ & \\
\hline $17 \mathrm{a}$ & 2.34 & dd & $15.3,5.0$ & 2.35 & dd & $14.0,5.5$ \\
\hline $17 b$ & 1.92 & $\mathrm{dd}$ & $14.4,9.2$ & 1.92 & dd & $14.0,9.5$ \\
\hline 18 & 2.16 & $\mathrm{~m}$ & & 2.17 & $\mathrm{~m}$ & \\
\hline 19 & 4.72 & $\mathrm{dd}$ & $6.1,3.2$ & 4.72 & dd & $5.9,3.4$ \\
\hline 20 & 2.85 & $\mathrm{dd}$ & $6.2,2.2$ & 2.85 & $\mathrm{dd}$ & $5.9,1.9$ \\
\hline 21 & 2.76 & $\mathrm{dd}$ & $7.5,2.2$ & 2.76 & $\mathrm{dd}$ & 1.9 \\
\hline 22 & $1.53-1.24$ & $\mathrm{~m}(5 \mathrm{H})$ & & 1.38 & $\mathrm{~m}$ & \\
\hline 23 & $1.53-1.24$ & $\mathrm{~m}(5 \mathrm{H})$ & & $1.55-1.25$ & $\mathrm{~m}(4 \mathrm{H})$ & \\
\hline 24 & $1.53-1.24$ & $m(5 \mathrm{H})$ & & $1.55-1.25$ & $\mathrm{~m}(4 \mathrm{H})$ & \\
\hline $25(3 \mathrm{H})$ & 0.91 & $\mathrm{t}$ & 7.1 & 0.91 & $\mathrm{t}$ & 6.8 \\
\hline $26(3 \mathrm{H})$ & 2.27 & $\mathrm{~d}$ & 1.1 & 2.27 & s & - \\
\hline $27 \mathrm{a}$ & 5.36 & $\mathrm{~d}$ & 1.0 & 5.36 & s & - \\
\hline $27 \mathrm{~b}$ & 5.21 & $\mathrm{~s}$ & - & 5.21 & $\mathrm{~s}$ & - \\
\hline $28 \mathrm{a}$ & 5.49 & $\mathrm{~s}$ & - & 5.49 & s & - \\
\hline $28 b$ & 5.38 & $\mathrm{~d}$ & 1.0 & 5.37 & s & - \\
\hline $29 a$ & 4.88 & $\mathrm{~s}$ & - & 4.88 & s & - \\
\hline $29 b$ & 4.79 & $\mathrm{~s}$ & - & 4.79 & s & - \\
\hline $30(3 \mathrm{H})$ & 1.06 & $\mathrm{~d}$ & 7.0 & 1.06 & $\mathrm{~d}$ & 7.0 \\
\hline $31(3 \mathrm{H})$ & 0.93 & $\mathrm{~d}$ & 6.8 & 0.93 & $\mathrm{~d}$ & 6.0 \\
\hline
\end{tabular}

Figure 10. ${ }^{1} \mathrm{H}$ NMR data for 11 in $\mathrm{CDCl}_{3}$ 


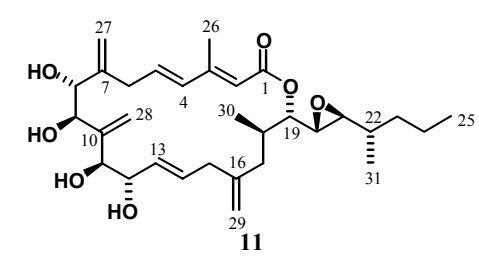

Isomer 11

$\underline{\text { Natural Product }}$

\begin{tabular}{|c|c|c|c|c|c|c|}
\hline Proton \# & $\delta_{\mathrm{H}}$ & Mult. & $J$ & $\delta_{\mathrm{H}}$ & Mult. & $J$ \\
\hline 2 & 5.83 & $\mathrm{~s}$ & - & 5.83 & $\mathrm{~s}$ & - \\
\hline 4 & 5.98 & $\mathrm{~d}$ & 15.6 & 5.98 & $\mathrm{~d}$ & 15.6 \\
\hline 5 & 5.71 & ddd & $14.9,8.9,5.6$ & 5.71 & ddd & $15.6,8.9,5.7$ \\
\hline $6 a$ & 2.88 & $\mathrm{~m}$ & & 2.87 & $\mathrm{~m}$ & \\
\hline $6 \mathrm{~b}$ & 2.88 & $\mathrm{~m}$ & & 2.87 & $\mathrm{~m}$ & \\
\hline 8 & 4.39 & $\mathrm{~s}$ br & - & 4.38 & $\mathrm{~s}$ br & - \\
\hline 9 & 4.45 & $\mathrm{~s}$ br & - & 4.44 & $\mathrm{~s}$ br & - \\
\hline 11 & 3.90 & $\mathrm{t}$ br & 3.9 & 3.89 & $\mathrm{~s}$ br & - \\
\hline 12 & 3.97 & $\mathrm{~s}$ br & - & 3.98 & $\mathrm{~s}$ br & - \\
\hline 13 & 5.29 & $\mathrm{dd}$ & $15.3,4.4$ & 5.29 & $\mathrm{dd}$ & $15.4,4.4$ \\
\hline 14 & 5.65 & ddd & $13.7,7.4,5.7$ & 5.65 & ddd & $15.4,7.4,6.5$ \\
\hline $15 \mathrm{a}$ & 2.63 & $\mathrm{dd}$ & $14.0,7.1$ & 2.63 & $\mathrm{dd}$ & $14.4,7.4$ \\
\hline $15 b$ & 2.56 & $\mathrm{dd}$ & $14.2,7.1$ & 2.56 & dd & $14.4,6.5$ \\
\hline $17 \mathrm{a}$ & 2.36 & $\mathrm{dd}$ & $14.5,4.4$ & 2.36 & $\mathrm{dd}$ & $14.7,6.2$ \\
\hline $17 \mathrm{~b}$ & 1.87 & $\mathrm{dd}$ & $14.4,8.8$ & 1.88 & $\mathrm{dd}$ & $14.7,8.9$ \\
\hline 18 & 2.19 & $\mathrm{~m}$ & & 2.19 & $\mathrm{~m}$ & \\
\hline 19 & 4.92 & $\mathrm{dd}$ & $6.8,3.1$ & 4.92 & $\mathrm{dd}$ & $6.8,3.1$ \\
\hline 20 & 2.79 & $\mathrm{dd}$ & $6.7,2.1$ & 2.79 & $\mathrm{dd}$ & $6.8,2.0$ \\
\hline 21 & 2.85 & $\mathrm{dd}$ & $7.6,2.0$ & 2.85 & $\mathrm{dd}$ & $7.5,2.0$ \\
\hline 22 & $1.48-1.12$ & $\mathrm{~m}(5 \mathrm{H})$ & & 1.24 & $\mathrm{~m}$ & \\
\hline 23 & $1.48-1.12$ & $\mathrm{~m}(5 \mathrm{H})$ & & $1.49-1.13$ & $\mathrm{~m}(4 \mathrm{H})$ & \\
\hline 24 & $1.48-1.12$ & $\mathrm{~m}(5 \mathrm{H})$ & & $1.49-1.13$ & $\mathrm{~m}(4 \mathrm{H})$ & \\
\hline $25(3 \mathrm{H})$ & 0.85 & $\mathrm{t}$ & 7.2 & 0.85 & $\mathrm{t}$ & 7.3 \\
\hline $26(3 \mathrm{H})$ & 2.21 & $\mathrm{~d}$ & 1.1 & 2.21 & $\mathrm{~s}$ & - \\
\hline $27 \mathrm{a}$ & 5.46 & $\mathrm{~s}$ & - & 5.45 & $\mathrm{~s}$ & - \\
\hline $27 \mathrm{~b}$ & 5.01 & $\mathrm{~s}$ br & - & 5.01 & $\mathrm{~s}$ br & - \\
\hline $28 \mathrm{a}$ & 5.43 & $\mathrm{t}$ & 1.3 & 5.42 & $\mathrm{t}$ & 1.3 \\
\hline $28 b$ & 5.15 & $\mathrm{~d}$ & 1.1 & 5.15 & $\mathrm{~d}$ & 1.3 \\
\hline $29 a$ & 4.86 & $\mathrm{~s}$ & - & 4.86 & $\mathrm{~s}$ & - \\
\hline $29 b$ & 4.78 & $\mathrm{~d}$ & 1.2 & 4.78 & $\mathrm{~d}$ & 1.5 \\
\hline $30(3 \mathrm{H})$ & 1.09 & $\mathrm{~d}$ & 7.0 & 1.09 & $\mathrm{~d}$ & 7.0 \\
\hline $31(3 \mathrm{H})$ & 0.83 & $\mathrm{~d}$ & 6.8 & 0.83 & $\mathrm{~d}$ & 6.8 \\
\hline
\end{tabular}

Figure 11. ${ }^{1} \mathrm{H}$ NMR data for 11 in $\mathrm{C}_{6} \mathrm{D}_{6}$ 


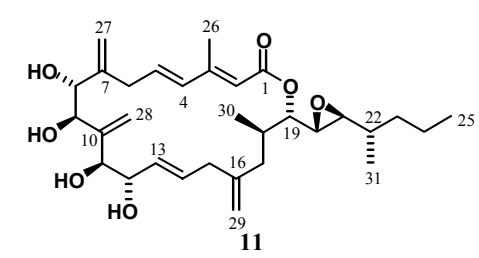

$\underline{\text { Isomer } 11}$

$\underline{\text { Natural Product }}$

\begin{tabular}{|c|c|c|c|c|c|c|}
\hline Proton \# & $\delta_{\mathrm{H}}$ & Mult. & $J$ & $\delta_{\mathrm{H}}$ & Mult. & $J$ \\
\hline 2 & 5.85 & $\mathrm{~s}$ & - & 5.85 & s & - \\
\hline 4 & 6.36 & d & 15.4 & 6.35 & d & 15.4 \\
\hline 5 & 6.14 & ddd & $15.1,8.9,5.9$ & 6.14 & ddd & $15.4,9.0,5.9$ \\
\hline $6 a$ & $3.12-3.09$ & $\mathrm{~m}(2 \mathrm{H})$ & & 3.10 & $\mathrm{~m}$ & \\
\hline $6 \mathrm{~b}$ & $3.12-3.09$ & $\mathrm{~m}(2 \mathrm{H})$ & & 3.10 & $\mathrm{~m}$ & \\
\hline 8 & 4.21 & $\mathrm{~s} \mathrm{br}$ & - & 4.21 & $\mathrm{~s} \mathrm{br}$ & - \\
\hline 9 & 4.46 & $\mathrm{~s} \mathrm{br}$ & - & 4.46 & $\mathrm{~s} \mathrm{br}$ & - \\
\hline 11 & 3.99 & $\mathrm{~d}$ br & 2.2 & 3.99 & d & 2.8 \\
\hline 12 & 4.13 & $\mathrm{~s} b r$ & - & 4.14 & $\mathrm{~s} \mathrm{br}$ & - \\
\hline 13 & 5.46 & $\mathrm{dd}$ & $15.3,5.3$ & 5.46 & $\mathrm{dd}$ & $15.6,5.5$ \\
\hline 14 & 5.59 & dddd & $15.3,7.7,6.2,1.5$ & 5.59 & ddd & $15.6,8.4,5.8$ \\
\hline $15 \mathrm{a}$ & 2.67 & $\mathrm{dd}$ & $14.5,7.9$ & 2.67 & $\mathrm{dd}$ & $14.3,8.4$ \\
\hline $15 \mathrm{~b}$ & 2.75 & $\mathrm{dd}$ & $14.3,6.2$ & 2.75 & $\mathrm{dd}$ & $14.3,5.8$ \\
\hline $17 \mathrm{a}$ & 2.41 & $\mathrm{dd}$ & $13.9,5.1$ & 2.41 & $\mathrm{dd}$ & $14.3,5.8$ \\
\hline $17 b$ & 1.92 & $\mathrm{dd}$ & $13.7,8.8$ & 1.92 & $\mathrm{dd}$ & $14.7,8.5$ \\
\hline 18 & 2.14 & $\mathrm{~m}$ & & 2.14 & $\mathrm{~m}$ & \\
\hline 19 & 4.69 & $\mathrm{dd}$ & $5.7,3.3$ & 4.69 & $\mathrm{dd}$ & $5.7,3.2$ \\
\hline 20 & 2.91 & $\mathrm{dd}$ & $5.7,2.2$ & 2.91 & $\mathrm{dd}$ & $5.7,2.2$ \\
\hline 21 & 2.75 & $\mathrm{dd}$ & $7.6,2.2$ & 2.75 & $\mathrm{dd}$ & $7.4,2.2$ \\
\hline 22 & $1.50-1.25$ & $\mathrm{~m}(5 \mathrm{H})$ & & 1.37 & $\mathrm{~m}$ & \\
\hline 23 & $1.50-1.25$ & $\mathrm{~m}(5 \mathrm{H})$ & & $1.50-1.25$ & $\mathrm{~m}(4 \mathrm{H})$ & \\
\hline 24 & $1.50-1.25$ & $\mathrm{~m}(5 \mathrm{H})$ & & $1.50-1.25$ & $\mathrm{~m}(4 \mathrm{H})$ & \\
\hline $25(3 \mathrm{H})$ & 0.91 & $\mathrm{t}$ & 7.1 & 0.91 & $\mathrm{t}$ & 7.2 \\
\hline $26(3 \mathrm{H})$ & 2.25 & d & 1.2 & 2.25 & $\mathrm{~s}$ & - \\
\hline $27 \mathrm{a}$ & 5.27 & s & 1.6 & 5.27 & $\mathrm{t}$ & 1.2 \\
\hline $27 \mathrm{~b}$ & 5.10 & $\mathrm{~s} \mathrm{br}$ & - & 5.10 & $\mathrm{~s} \mathrm{br}$ & - \\
\hline $28 \mathrm{a}$ & 5.40 & $\mathrm{t}$ & 1.5 & 5.40 & $\mathrm{t}$ & 1.3 \\
\hline $28 b$ & 5.34 & $\mathrm{~s}$ & - & 5.35 & $\mathrm{~s} \mathrm{br}$ & - \\
\hline $29 a$ & 4.85 & s & - & 4.85 & $\mathrm{~s}$ & - \\
\hline $29 b$ & 4.76 & $\mathrm{~s} \mathrm{br}$ & - & 4.76 & $\mathrm{~s} \mathrm{br}$ & - \\
\hline $30(3 \mathrm{H})$ & 1.06 & d & 7.1 & 1.06 & d & 7.1 \\
\hline $31(3 \mathrm{H})$ & 0.92 & d & 6.7 & 0.92 & d & 6.8 \\
\hline
\end{tabular}

Figure 12. ${ }^{1} \mathrm{H}$ NMR data for 11 in $\mathrm{CD}_{3} \mathrm{OD}$ 


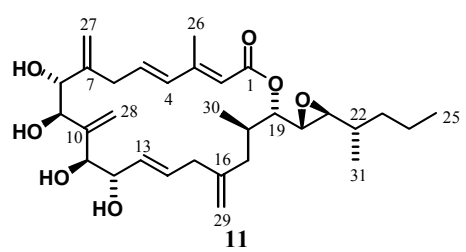

\begin{tabular}{|c|c|c|}
\hline & $\underline{\text { Isomer } 11}$ & $\underline{\text { Natural Product }}$ \\
\hline Carbon \# & $\delta_{\mathrm{C}}$ & $\delta_{\mathrm{C}}$ \\
\hline 1 & 165.8 & 165.8 \\
\hline 2 & 118.6 & 118.6 \\
\hline 3 & 152.7 & 152.8 \\
\hline 4 & 136.3 & 136.4 \\
\hline 5 & 134.7 & 134.8 \\
\hline 6 & 39.0 & 39.0 \\
\hline 7 & 144.8 & 144.8 \\
\hline 8 & 72.5 & 72.5 \\
\hline 9 & 70.6 & 70.5 \\
\hline 10 & 147.2 & 147.2 \\
\hline 11 & 75.8 & 75.8 \\
\hline 12 & 73.5 & 73.5 \\
\hline 13 & 130.5 & 130.6 \\
\hline 14 & 130.9 & 130.9 \\
\hline 15 & 39.7 & 39.7 \\
\hline 16 & 145.0 & 145.0 \\
\hline 17 & 36.2 & 36.2 \\
\hline 18 & 33.3 & 33.3 \\
\hline 19 & 74.7 & 74.7 \\
\hline 20 & 54.2 & 54.2 \\
\hline 21 & 61.8 & 61.8 \\
\hline 22 & 35.3 & 35.4 \\
\hline 23 & 36.7 & 36.7 \\
\hline 24 & 19.9 & 20.0 \\
\hline 25 & 14.2 & 14.3 \\
\hline 26 & 13.9 & 13.9 \\
\hline 27 & 114.6 & 114.7 \\
\hline 28 & 116.0 & 116.1 \\
\hline 29 & 112.8 & 112.9 \\
\hline 30 & 14.9 & 14.9 \\
\hline 31 & 15.8 & 15.8 \\
\hline
\end{tabular}

Figure 13. ${ }^{13} \mathrm{C}$ NMR data for 11 in $\mathrm{CDCl}_{3}$ 


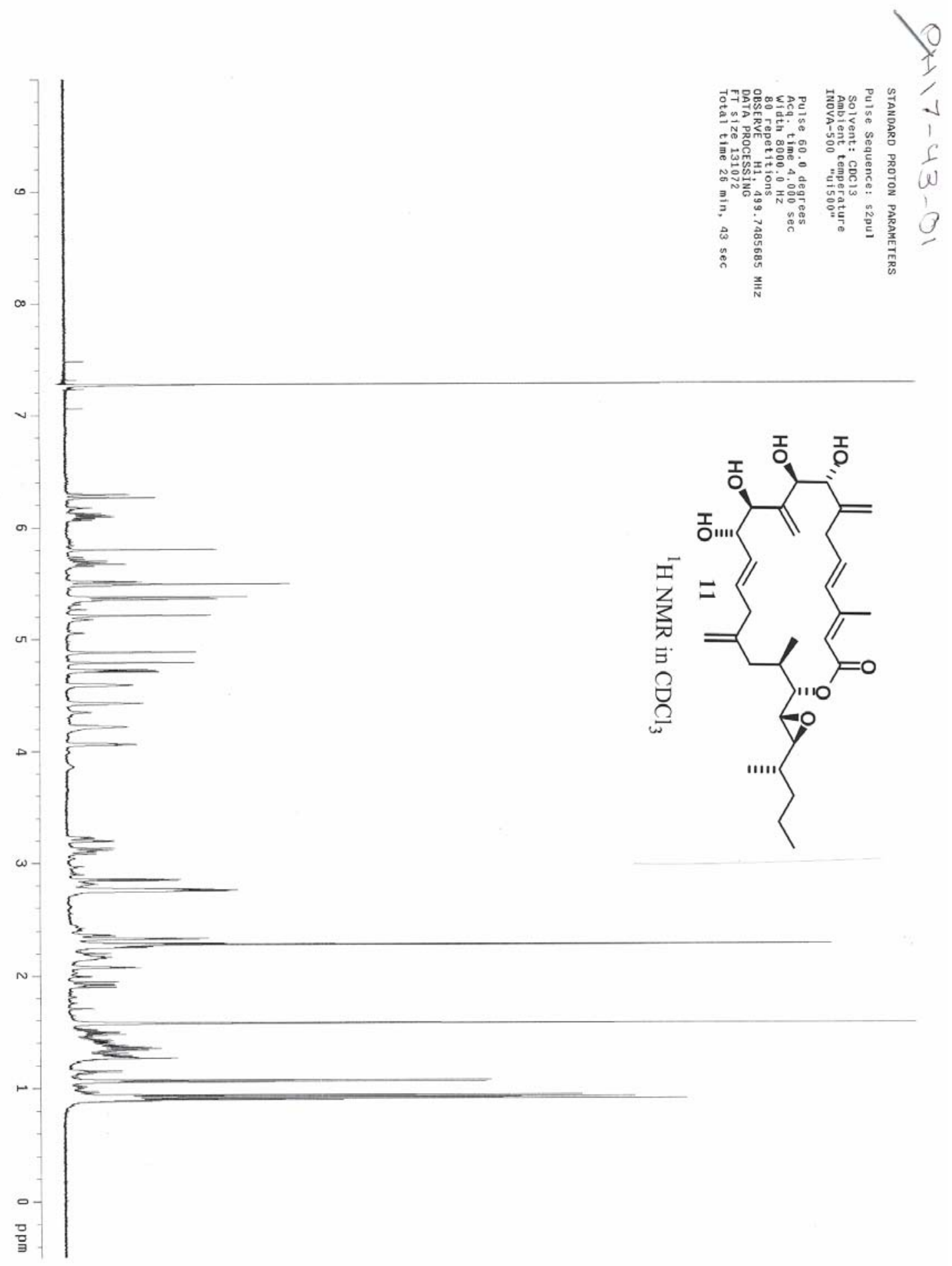




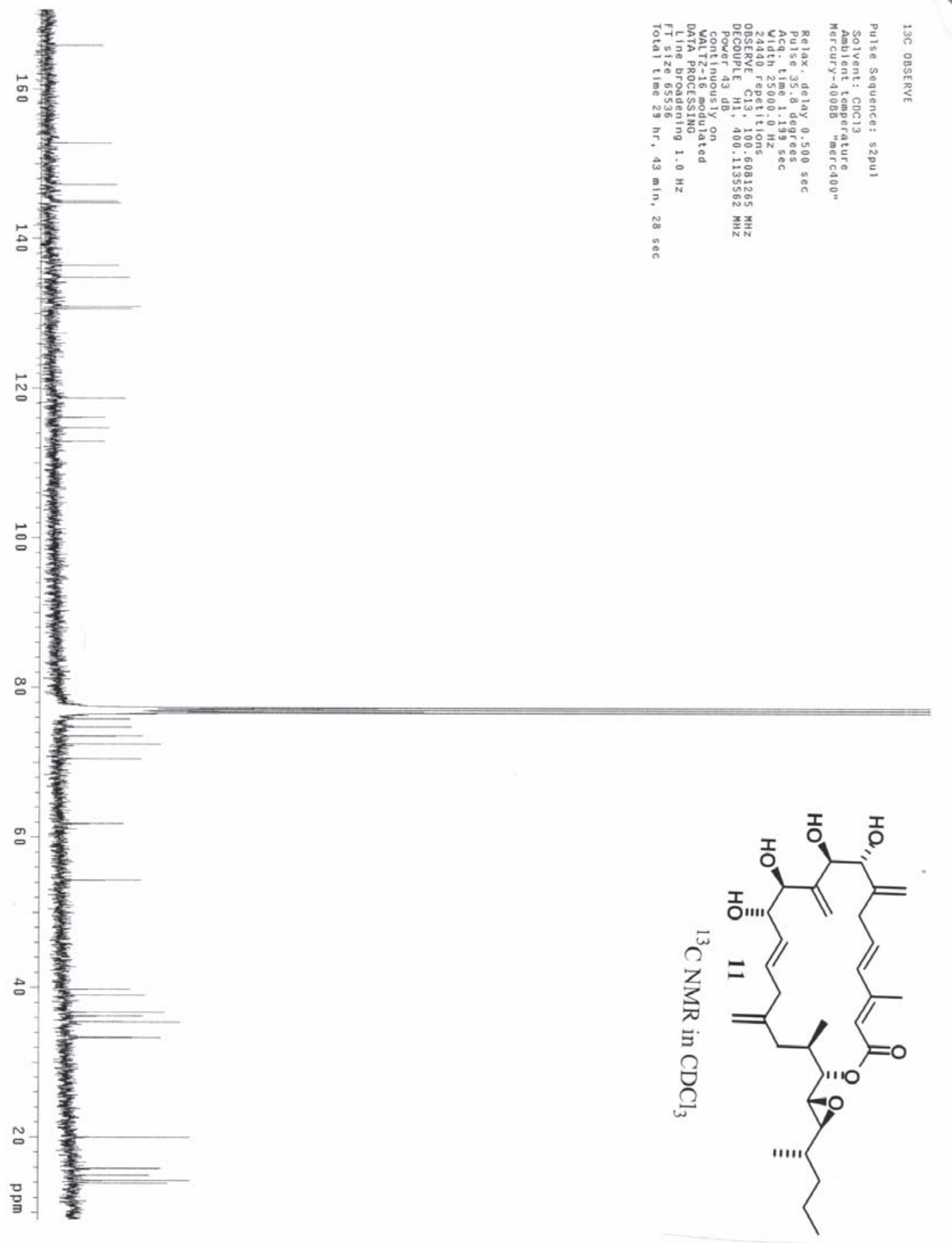

